\title{
Nudging als politisches Instrument - gute Absicht oder staatlicher Übergriff?
}

Article

Accepted Version

Bruttel, L. V., Stolley, F., Guth, W., Kliemt, H., Bosworth, S., Bartke, S., Schnellenbach, J., Weimann, J., Haupt, M. and Funk, L. (2014) Nudging als politisches Instrument - gute Absicht oder staatlicher Übergriff? Wirtschaftsdienst, 94 (11). pp. 767-791. ISSN 1613-978X doi:

https://doi.org/10.1007/s10273-014-1748-9 Available at https://centaur.reading.ac.uk/72601/

It is advisable to refer to the publisher's version if you intend to cite from the work. See Guidance on citing.

To link to this article DOI: http://dx.doi.org/10.1007/s10273-014-1748-9

Publisher: Springer

All outputs in CentAUR are protected by Intellectual Property Rights law, including copyright law. Copyright and IPR is retained by the creators or other copyright holders. Terms and conditions for use of this material are defined in the End User Agreement.

www.reading.ac.uk/centaur

\section{CentAUR}


Central Archive at the University of Reading

Reading's research outputs online 


\title{
Nudging als politisches Instrument - gute Absicht oder staatlicher Übergriff?
}

\author{
Die Verhaltensökonomie und ihre praktischen Implikationen geraten immer stärker in den \\ Fokus auch der deutschen Politik. Individuelle Entscheidungen sollen im Sinne eines „libertären \\ Paternalismus“ „sanft“ beeinflusst werden. Die „Nudges“ bestehen in Standardvorgaben, \\ Selbstbindungen und der Informationsbereitstellung. Deren Anwendung setzt allerdings die \\ Klärung einiger Fragen voraus: Wer darf sich anmaßen, für die Wirtschaftssubjekte „kluge“ \\ Entscheidungen zu treffen? Bei welchen Entscheidungen sind Eingriffe einer anderen Instanz \\ begründbar? Mit welchem Zeithorizont und aufgrund welcher Wohlfahrtsüberlegungen wird eine \\ Entscheidung als „richtig“ definiert? Welcher Rationalitätsbegriff steht hinter dem Konzept?
}

Lisa V. Bruttel, Florian Stolley

\section{Ist es im Interesse der Bürger, wenn ihre Regierung Nudges implementiert?}

Nicht erst seit dem Buch „Nudge - Wie man kluge Entscheidungen anstößt" von Richard Thaler und Cass Sunstein ist bekannt, ${ }^{1}$ dass Menschen Schwierigkeiten haben, für sich selbst optimale Entscheidungen zu treffen. Sie treiben weniger Sport und essen ungesünder, als sie es sich vorgenommen haben, sie legen zu wenig Geld für das Alter zurück, und sie schieben unangenehme Aufgaben und Entscheidungen auf, länger als innen selbst lieb ist. Zudem hat die experimentelle Wirtschaftsforschung wiederholt gezeigt, dass Menschen selbst bei einer vermeintlich rationalen Beurteilung von Entscheidungsalternativen systematische Fehler unterlaufen. ${ }^{2}$ Nach Thaler und Sunstein kann dieser Mangel an Selbstkontrolle und Rationalität durch sogenannte „Nudges“ ausgeglichen werden. Die ldee dabei ist, durch eine behutsame, quasi „minimalinvasive“ Umgestaltung der Entscheidungssituation z.B. komplexe Entscheidungen zu erleichtern oder Selbstkontrollprobleme zu reduzieren, ohne dabei de facto die Entscheidungsfreiheit zu beschneiden. Viele Menschen sind, so Thaler und Sunstein, ehrlich dankbar für gut konstruierte Nudges, etwa das Platzieren von gesundem Essen auf Augenhöhe in einer Cafeteria, was den Verzehr ungesunder Speisen nur unwesentlich schwerer macht. Werden solche Nudges von staatlicher Seite eingesetzt, sprechen Thaler und Sunstein daher von einem Konzept des „libertären Paternalismus“, im Gegensatz zu „echtem“ Paternalismus, wie es das Verbieten von ungesundem Essen wäre. Beispiele für Situationen, in denen sich Individuen nicht in ihrem eigenen

1 R. H. Thaler, C. R. Sunstein: Nudge - Wie man kluge Entscheidungen anstößt, Berlin 2009.

2 Vgl. hierzu unter anderem die vielen Beiträge von Daniel Kahneman und Amos Tversky, zusammengefasst in: D. Kahneman: Schnelles Denken, langsames Denken, München 2012.
Interesse verhalten und in denen Nudges helfen können, sind zahlreich. Eine Auswahl wollen wir zunächst näher vorstellen:

\section{Defaults}

Die bekannteste Art von Nudges sind sicherlich Standardvorgaben, die Defaults. Diese sollen Menschen in eine empfehlenswerte Richtung „stupsen“, indem sie in Entscheidungssituationen einen optimalen Standard als Vorauswahl anbieten. Thaler und Sunstein beschreiben in ihrem Buch einige Beispiele, in denen deutlich wird, wie stark Standardvorgaben die Entscheidung von Individuen beeinflussen. Das bekannteste und am meisten zitierte Beispiel ist hierbei die Teilnahme am System der betrieblichen Altersvorsorge in den USA. Wenn der Beitritt zur betrieblichen Altersvorsorge automatisch erfolgt und ein Arbeitnehmer, der das nicht möchte, dem Beitritt aktiv widersprechen muss, entscheiden sich signifikant mehr Arbeitnehmer für eine betriebliche Altersvorsorge als im umgekehrten Fall. ${ }^{3}$ In Deutschland stellt sich diese Frage allerdings kaum, weil das hiesige Rentensystem im Vergleich zum US-amerikanischen eine deutlich bessere Altersvorsorge sicherstellt - übrigens eine Form von Paternalismus, die zu Recht kaum infrage gestellt wird. ${ }^{4}$

3 Vgl. B. Madrian, D. Shea: The Power of Suggestion: Inertia in 401(k) Participation and Savings Behavior, in: The Quarterly Journal of Economics, 116. Jg. (2001), H. 4, S. 1149-1187.

4 Als weiteres Beispiel für einen Default-Nudge, für den die meisten Bürger sehr dankbar seien, nennen Thaler und Sunstein die Zeitumstellung im Sommer, die den Vorsatz, in der warmen Jahreszeit früher aufzustehen, leichter umsetzbar macht. Für $67 \%$ der Deutschen, die die Sommerzeit am liebsten abschaffen würden, vgl. O.V.: Hohles Versprechen, in: Stern, Nr. 14 vom 27.3.2014, S. 21, ist das allerdings eindeutig zu viel des Paternalismus. 


\section{Selbstbindung}

Nudges sind aber noch viel mehr als die bekannten Defaults. Bei den eingangs genannten Beispielen mangelnder Selbstkontrolle liegt der Kern des Problems oft in zeitinkonsistentem Verhalten. ${ }^{5}$ Die Entscheidung, Sport zu treiben, und damit die Abwägung zwischen den „Kosten“ (Anstrengung, Opportunitätskosten der Zeit) und dem „Nutzen“ (Fitness, Gesundheit) wird zu Neujahr alljährlich langfristig optimal getroffen. Steht jedoch die Entscheidung an, genau jetzt zum Sport zu gehen, ist das Optimalitätskalkül ein anderes, da die „Kosten“ direkt anfallen und unmittelbar als relativ hoch empfunden werden, während der langfristige „Nutzen“ erst verzögert eintritt und damit im momentanen Optimalitätskalkül tendenziell untergewichtet wird. Bei der Bewältigung dieser Selbstkontrollprobleme können Nudges, die die Anreizstruktur verändern - von staatlicher oder privater Seite angeboten - behilflich sein. Vergleichsweise gut funktionieren z.B. Wetten mit anderen Personen, ob man es schafft, in Zukunft in bestimmter Weise zu handeln (z.B. mit dem Rauchen aufzuhören). Solch eine Wette hat, wenn man sie verliert, direkte Konsequenzen, und zwar soziale (das Eingestehen von Scheitern gegenüber einem Bekannten) sowie möglicherweise auch finanzielle (die Übergabe des Gewinns an den Bekannten). Die Konsequenzen einer Wette ändern also die Anreize, wodurch sich das Verhalten wandeln kann. ${ }^{6}$ Diese Art der Selbstbeschränkung wird komplett frei gewählt, so dass die Entscheidungsfreiheit ex ante gewährt bleibt. In gewisser Hinsicht sind solche Selbstkontroll-Nudges daher harmloser als die bekannteren Defaults.

\section{Information}

Nudges können des Weiteren Entscheidungen von Menschen verbessern, wenn sie in Form von gut aufbereiteter Information daherkommen. Wie insbesondere durch Kahneman und Tversky seit den 1970er Jahren dokumentiert, ${ }^{7}$ lassen verschiedene kognitive Verzerrungen sowie falsch angewendete Faustregeln Menschen oft irrationale Entscheidungen treffen. Ohne diese Verzerrungen und Faustregeln detailliert zu beschreiben, sei zumindest erwähnt, dass Menschen vor allem mit dem Einschätzen und Analysieren von komplexen und seltenen Entscheidungssituationen Schwierigkeiten haben. Nudges können hier helfen, indem sie es Personen erleichtern, die wichtigen und zentralen Informationen herauszufiltern, was ein vereinfachtes Einschätzen

5 Vgl. hierzu unter anderem D. Read, B. Leeuwen: Predicting Hunger: The Effects of Appetite and Delay on Choice, in: Organizational Behavior and Human Decision Processes, 76. Jg. (1998), Nr. 2, S. 189-205.

6 Eine ähnliche Wirkung haben z.B. Computerprogramme, mit denen man bestimmte Webseiten für eine vorher eingestellte Zeit blockieren kann, um effizienter zu arbeiten, und die vermutlich schon mehreren Studierenden die fristgerechte Abgabe ihrer Abschlussarbeit gerettet haben.

7 D. Kahneman, a.a.O. und Vergleichen von verschiedenen Alternativen ermöglicht. Beispielsweise sind im Versicherungsbereich genaue Informationen über Wahrscheinlichkeiten und Schadenssummen nötig, um rationale Entscheidungen zu treffen. Hier können Informations-Nudges, die diese Informationen in übersichtlicher Form - möglicherweise sogar individualisiert auf die jeweilige Situation - zur Verfügung stellen, helfen. ${ }^{8}$

Auch wenn man Nudges also durchaus als sehr nützlich ansehen kann, herrscht in der öffentlichen Diskussion teilweise Skepsis gegenüber staatlich eingesetzten Nudges. Die Kritik reicht vom Vorwurf der Bevormundung der Bürger in privaten Angelegenheiten bis hin zur mutmaßlichen Ausübung von Zwang durch Nudges. ${ }^{9}$ Diese Skepsis mag im Licht einer durchaus beobachtbaren paternalistischen Grundtendenz in der deutschen beziehungsweise europäischen Politik berechtigt erscheinen. Während Thaler und Sunstein noch zurückhaltend vorschlagen, intelligente Lampen und Kabel einzuführen, die Verbrauchern ihren Stromverbrauch plastisch zeigen und dadurch den Verbrauch reduzieren sollen, verbietet die Europäische Union den Verkauf von Glühbirnen. ${ }^{10}$ Als weitere Beispiele für paternalistische Tendenzen in der Politik lassen sich Rauchverbote auf Bundes-11 oder auf Landesebene $^{12}$ in Deutschland anführen oder die Pflicht zu Kindervorsorgeuntersuchungen in manchen Bundesländern, die infolge von publik gewordenen Fällen von verwahrlosten oder misshandelten Kindern eingeführt wurde.

Um es klarzustellen: Wir sind keineswegs Gegner dieser Maßnahmen. Es soll daran nur deutlich gemacht werden, dass sich in Deutschland und der EU gegenwärtig eine Tendenz zu paternalistischer Politik beobachten lässt und dass diese Tendenz die Skepsis, mit der der Einsatz von Nudges betrachtet wird, möglicherweise noch vergrößert. ${ }^{13}$ Die Befürchtung, Nudges könnten seitens der Regierung eingesetzt werden, um Bürger unterbewusst - auch gegen ihren Willen - zu beeinflussen, und der Staat könne sich durch den

8 Neben einer besser informierten Entscheidung von Individuen ließen sich diese Nudges auch dazu benutzen, nur bestimmte Informationen deutlicher und damit präsenter zu machen und dadurch die individuelle Entscheidung in eine vom Staat gewünschte Richtung zu beeinflussen. Dies entspricht in vielen Aspekten dem, was Firmen seit jeher mit Werbung zu erreichen versuchen, und geht wieder stärker in die Richtung eines reinen Paternalismus.

9 P. Plickert, H. Beck: Kanzlerin sucht Verhaltensforscher, http://ow.ly/ DQsSj (26.8.2014); P. Plickert: Die große Bevormundung durch die Verhaltensökonomen, 2012, http://ow.ly/DQsY6 (16.3.2012).

10 Verordnung (EG) Nr. 244/2009 der Kommission vom 18.3.2009 zur Durchführung der Richtlinie 2005/32/EG des Europäischen Parlaments und des Rates im Hinblick auf die Festlegung von Anforderungen an die umweltgerechte Gestaltung von Haushaltslampen mit ungebündeltem Licht.

11 Gesetz zur Einführung eines Rauchverbotes in Einrichtungen des Bundes und öffentlichen Verkehrsmitteln (Bundesnichtraucherschutzgesetz - BnichtrSchG) vom 20.7.2007.

12 Eine Übersicht über die einzelnen Rauchverbote in den Bundesländern findet sich hier: http://ow.ly/DQtoC (21.10.2014).

13 K. Horn: Sklavenhalter der Zukunft, http://ow.ly/DQt7f (11.3.2013). 


\section{Die Autoren des Zeitgesprächs}

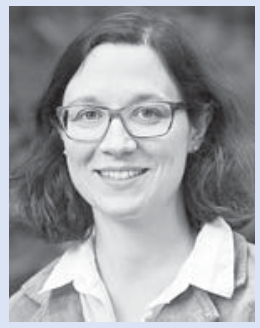

Prof. Dr. Lisa V. Bruttel lehrt Volkswirtschaftslehre, insbesondere Märkte, Wettbewerb und Institutionen an der Universität Potsdam.

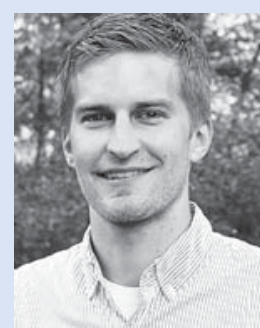

Florian Stolley, M.Sc., ist wissenschaftlicher Mitarbeiter am Lehrstuhl für Volkswirtschaftslehre der Universität Potsdam.

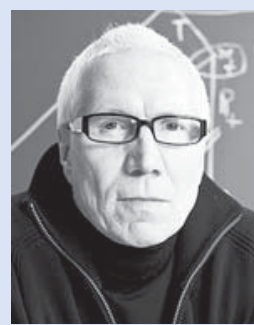

Prof. Dr. Werner Güth ist Direktor der Strategic Interaction Group des Max-Planck-Instituts für Ökonomik in Jena.

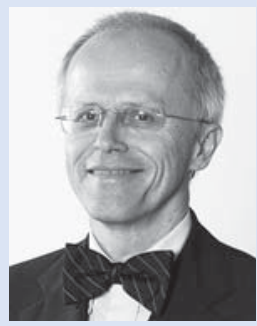

Prof. Dr. Hartmut Kliemt ist Vizepräsident Forschung und Professor für Philosophy \& Economics der Frankfurt School of Finance \& Management.

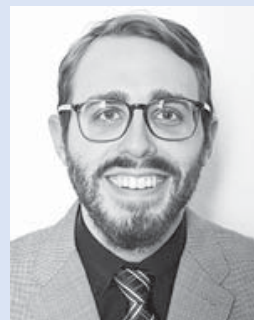

Steven Bosworth, Ph.D, ist PostDoktorand am Institut für Weltwirtschaft in Kiel.

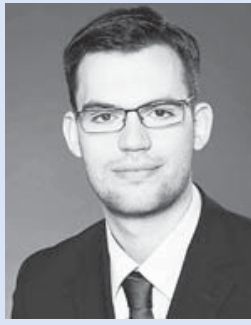

Simon Bartke, M.Sc., ist wissenschaftlicher Mitarbeiter am Institut für Weltwirtschaft in Kiel.

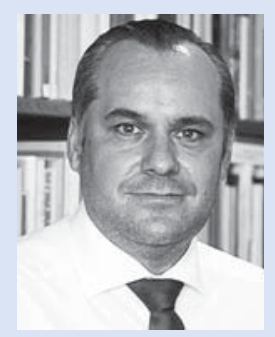

Prof. Dr. Jan Schnellenbach lehrt Volkswirtschaftslehre, insbesondere Mikroökonomik an der Brandenburgischen Technischen Universität Cottbus-Senftenberg.

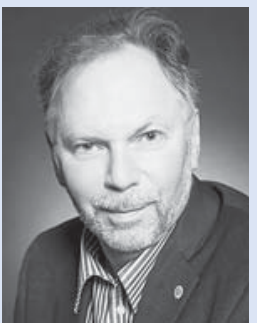

Prof. Dr. Joachim Weimann lehrt Wirtschaftspolitik an der Otto-vonGuericke-Universität in Magdeburg

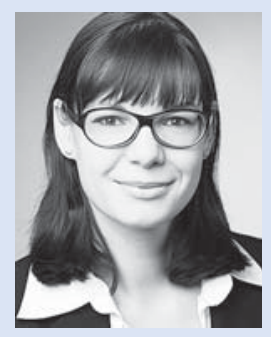

Dr. Marlene Haupt ist wissenschaftliche Mitarbeiterin am Max-PlanckInstitut für Sozialrecht und Sozialpolitik in München.

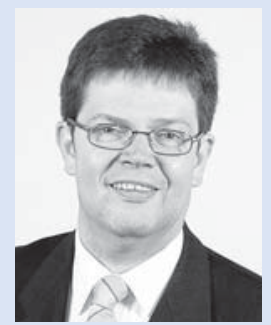

Prof. Dr. Lothar Funk lehrt Volkswirtschaftslehre und internationale Wirtschaftsbeziehungen an der Fachhochschule Düsseldorf. 
Einsatz von Nudges zunehmend in private Angelegenheiten einmischen, erscheint jedenfalls durchaus berechtigt. ${ }^{14}$ In bestimmten Entscheidungssituationen, die z.B. ethisch besonders kontrovers sind, fühlen sich viele Bürger zu Recht unwohl, wenn ihre Regierung versucht, Entscheidungen zu beeinflussen. Als Beispiel sei hier nur die kürzlich wieder aufgeflammte Diskussion um den Mangel an gespendeten Organen genannt, und die Frage, ob eine Widerspruchslösung, die zu einer dramatisch höheren Zahl an Organspendern führen würde, ${ }^{15}$ anstelle der derzeit geltenden Zustimmungslösung moralisch vertretbar wäre. ${ }^{16}$

Das Beispiel der Organspende zeigt, dass der Einsatz von Nudges durch den Staat behutsam und transparent erfolgen muss und dass besondere Aufmerksamkeit der Frage gelten sollte, welchen Bereich diese Entscheidungen tangieren. Eine Nicht-Beachtung dieser Prinzipien würde die Kritik am Einsatz von Nudges generell verstärken. Dabei gibt es genügend Anwendungsbeispiele, in denen der sinnvolle und transparente Einsatz von Nudges von nahezu allen Seiten unterstützt wird. Eine pauschale Verteufelung oder Lobpreisung ist daher nicht zielführend. Nudges müssen differenzierter nach Art und Zweck bewertet werden.

\section{Informations-Nudges}

Ein breiter Konsens für den Einsatz von staatlichen Nudges ist insbesondere zu erwarten, wenn ein Nudge das Ziel hat, die Bürger mündiger in ihrer Entscheidung zu machen, indem innen mehr Informationen zur Verfügung gestellt werden, und sie daher eine besser fundierte Entscheidung treffen können. Dabei lassen sich Nudges in Form der Bereitstellung von Informationen grob in zwei Gruppen untergliedern: Informationen, die dem Schutz schwächerer Marktteilnehmer dienen, und Informationen, die das Verhalten in eine (individuell und gesellschaftlich) wünschenswerte Richtung lenken sollen.

Informationsbereitstellung zum Schutz schwächerer Marktteilnehmer ist dann sinnvoll, wenn in Entscheidungssituationen relevante Informationen, die einen einfachen Vergleich der Alternativen ermöglichen, nicht leicht zu bekommen sind. Insbesondere für komplexe Entscheidungen kann der Staat klare Regeln vorgeben, welche Informationen der stärkere beziehungsweise besser informierte Marktteilnehmer dem schwächeren beziehungsweise schlechter informierten Marktteilnehmer in welcher Form zukommen lassen muss.

14 Vgl. J. Schnellenbach: Wohlwollendes Anschubsen: Was ist mit liberalem Paternalismus zu erreichen und was sind seine Nebenwirkungen?, in: Perspektiven der Wirtschaftspolitik, 12. Jg. (2011), H. 4, S. 445-459.

15 Vgl. E. Johnson, D. Goldstein: Do Defaults Save Lives?, in: Science, 302. Jg. (2003), S. 1338-1339.

16 O.V.: Zahl der Organspender fällt auf historisches Tief, http://ow.ly/DQrBI (15.1.2014); M. Bartsch: Gesetzesvorschlag: Minister wollen jeden Deutschen zum Organspender erklären, http://ow.ly/DQrlU (1.6.2011).
Ein bisher nicht erfolgreich etablierter, aber sehr vielversprechender Vorschlag für einen solchen Informations-Nudge, den Verbraucherschützer energisch befürworteten, ${ }^{17}$ ist die Lebensmittel-Ampel. Diese sollte es dem Verbraucher erleichtern, gesundheitsrelevante Informationen über die Inhaltsstoffe von Lebensmitteln zu bekommen. Die Kennzeichnung galt jedoch als zu stark vereinfachend und in Teilen irreführend, sodass sich die Europäische Union vorerst gegen die verpflichtende Einführung einer solchen LebensmittelAmpel ausgesprochen hat.18

Informations-Nudges mit dem Ziel einer Verhaltenslenkung können unter anderem im Bereich des Energieverbrauchs sinnvoll sein. Wie bereits erwähnt, hat sich die EU gegen einen Nudge und für ein „klassisches“ Verbot entschieden, um die Nutzung von Glühbirnen, die viel Energie verbrauchen, zu reduzieren. Wir glauben, dass der Vorschlag von Thaler und Sunstein, Bürgern ein direktes Feedback über ihren Stromverbrauch zu geben, das Verhalten positiv beeinflussen kann. Stromverbrauch ist etwas sehr Abstraktes und eine jährliche Abrechnung versetzt kaum einen Menschen in die Lage, die Kosten des alltäglichen Verbrauchs richtig einzuschätzen. Die Installation intelligenter Stromzähler in privaten Haushalten, die nicht nur den Verbrauch sinnvoll steuern können, ${ }^{19}$ sondern auch direktes, leicht wahrnehmbares Feedback über den alltäglichen Energieverbrauch bereitstellen, würde den Verbraucher in die Lage versetzten, seinen Stromverbrauch zu kontrollieren und Einsparpotenziale zu realisieren, ohne ihm irgendwelche Vorschriften zu machen. ${ }^{20}$

\section{Selbstkontroll-Nudges}

Für unbedenklich halten wir auch eine behutsame staatliche Unterstützung im Hinblick auf Selbstkontrollprobleme. Ein Beispiel für einen bereits vom Staat eingeführten Nudge ist die Selbstsperre beim Glücksspiel in Spielbanken in Deutschland. Neben staatlichen Angeboten zur Hilfe bei Spielsucht können Spieler, die befürchten, in der Zukunft die Kontrolle über sich selbst zu verlieren, einen Antrag auf Selbstsperre stellen, wodurch innen der Zutritt zu Spielbanken deutschlandweit ver-

17 B. Weitzel: Kunden wählen Rot-Gelb-Grün, http://ow.ly/DQqMS (16.6.2009).

18 D. Kuhr: Aigner lehnt Lebensmittel-Ampel ab, http://ow.ly/DQr8g (3.7.2013).

19 O.V.: Bund sagt intelligenten Stromzählern ade, http://ow.ly/DQrgo (1.10.2014).

20 Häufig werden solche Informations-Nudges auch im Gesundheitsbereich angewendet. Der Staat versucht hierdurch Gesundheitsrisiken deutlich zu machen und das Verhalten im Hinblick auf diese Risiken zu ändern. Die Bundeszentrale für gesundheitliche Aufklärung (BZgA) stellt z.B. Informationen zum richtigen Verhalten während der Schwangerschaft und zum Umgang mit Kleinkindern, die werdende Eltern an verschiedenen Stellen angeboten bekommen, bereit. Beispielsweise gibt es Kampagnen gegen das Rauchen während der Schwangerschaft oder solche, die auf die Gefahren des Schüttelns von Babys hinweisen (siehe www.kindergesundheit-info.de oder www.familienplanung.de). 
wehrt wird. ${ }^{21}$ Eine Selbstsperre ist vollkommen freiwillig, somit ist die Entscheidungsfreiheit des Individuums gewahrt.

Weiterhin könnten wir uns vorstellen, dass der Staat - beispielsweise in Gestalt der Bundeszentrale für gesundheitliche Aufklärung (BZgA) - den Aufbau einer Internet-Plattform unterstützt, die es interessierten Bürgern erleichtert, Vorsätze - wie z.B. mit dem Rauchen aufzuhören oder mehr Sport zu treiben - umzusetzen. Als Vorbild könnte hier die privat geführte, amerikanische Internetseite StickK.com dienen, die es ihren Nutzern ermöglicht, bindende Verträge zur Lösung von Selbstkontrollproblemen zu schließen. ${ }^{22}$ Hierbei wird ein Vorhaben spezifiziert, ein Bekannter benannt, der überprüft, ob das Vorhaben erfolgreich geschafft wurde, und ein Geldbetrag gesperrt, den der Teilnehmer nach erfolgreichem Umsetzen des Vorsatzes wiederbekommt. Sollte das Vorhaben nicht geschafft werden, geht das Geld an eine vorher festgelegte Institution oder Person. Der Wunsch, das Geld nicht zu verlieren sowie kein Scheitern gegenüber Freunden eingestehen zu müssen, dient erfolgreich als Motivation, den Vorsatz tatsächlich umzusetzen. Ähnlich wie die Selbstsperre beim Glücksspiel wäre die Teilnahme an diesem Angebot natürlich vollkommen freiwillig - und ein staatlicher Betrieb der Internetseite möglicherweise sogar vertrauenswürdiger als ein privater Anbieter.

\section{Schutz vor dem benachteiligenden kommerziellen Einsatz von Nudges}

Neben einem möglichen aktiven Einsatz von Nudges kommt dem Staat noch eine wichtige Rolle beim Schutz seiner Bürger vor Ausbeutung durch den kommerziellen Einsatz von Nudges, insbesondere im Bereich der Default-Nudges, zu. Denn der Staat hat nicht nur die Möglichkeit Standardvorgaben setzen, sondern auch bestimmte zu verbieten, um

21 §8 Spielersperre, Staatsvertrag zum Glücksspielwesen in Deutschland (Glücksspielstaatsvertrag - GlüStV) vom 15.12.2011.

22 Diese Plattform wurde von Professoren der Universität Yale gegründet.
z.B. schwächere Marktteilnehmer zu schützen. Hier sind in Deutschland und Europa schon eine Reihe von sinnvollen Maßnahmen umgesetzt worden. Neben einer verbraucherfreundlichen Gesetzeslage bei Fernabsatzverträgen, die das Ausnutzen von Informationsvorteilen der Händler verhindern soll, wurde es Händlern beispielweise untersagt, einem Online-Kunden den Bezug eines Newsletters per DefaultHäkchen unterzujubeln. ${ }^{23}$ Ähnlich verhält es sich bei Flugbuchungen im Internet, bei denen der Kauf einer Zusatzversicherung nicht voreingestellt sein darf. ${ }^{24}$

\section{Fazit}

Nudges sind mehr als nur das Setzen von Defaults. Gute Nudges können Selbstkontrollprobleme reduzieren oder durch das Bereitstellen von Informationen Entscheidungen verbessern und erleichtern. Beim staatlichen Einsatz von Nudges besteht jedoch eine gewisse Skepsis, da man bestimmte Varianten auch als Manipulation und Bevormundung verstehen kann. Daher plädieren wir dafür, dass ihr Einsatz behutsam und transparent erfolgen muss und dass eine Diskussion nötig ist, für welche Bereiche sich die Anwendung eines Nudges rechtfertigen lässt. Weitgehend unstrittig ist sicherlich die Sichtweise, dass der Staat seine Bürger vor dem benachteiligenden Einsatz von kommerziellen Nudges schützen sollte, indem er die Bereitstellung von gut verständlichen Informationen vorschreibt oder regulierend in die Nutzung von Defaults eingreift. Daneben gibt es Einsatzbereiche, in denen wir es für unbedenklich, wenn nicht sogar wünschenswert halten, dass staatliche Stellen selbst aktiv Nudges konstruieren. Dies umfasst insbesondere jene Nudges, die Bürgern übersichtliche Informationen zur Verfügung stellen (etwa zu ihrem Stromverbrauch) und sie dadurch in ihren Entscheidungen mündiger machen, sowie Nudges, die Bürgern unterstützende Angebote machen, ihre Selbstkontrolle in den Griff zu bekommen, wenn sie selbst diesen Vorsatz fassen.

23 Bundesgerichtshof, Urteil vom 16.7.2008, VIII ZR 348/06.

24 Landgericht Frankfurt am Main, Urteil vom 22.1.2014, Az. 2-06 O 379/13.

Werner Güth, Hartmut Kliemt

\section{Nudging: Obrigkeitsdenken und Verwaltungsfreude in neuem Gewande?}

In „Asterix bei den Goten“ “ finden wir Ovids Sentenz „video meliora proboque deteriora sequor" (ich sehe das bessere und ich heiße es gut, dem schlechteren werde ich nachgehen). Walter Mischel benutzte seinen Marshmallow-Test Kinder, die der süßen Versuchung widerstehen müssen, um danach die doppelte Menge zu bekommen - ursprünglich

1 R. Goscinny, A. Uderzo: Die Abenteuer von Asterix der Gallier - Asterix bei den Goten, Bd. 3, Paris 1963 oder Berlin 1970, S. 20. nicht zu diagnostischen Zwecken, ${ }^{2}$ sondern um herauszufinden, wie bereits Kinder sich gegen ihre „zu große“ Schwäche für das Naheliegende gegenüber dem Fernliegenden wappnen. Diese Strategien im Umgang mit Emotionen sind das Interessante, nicht die Tatsache, dass wir Versuchungen unterliegen. Odysseus lässt sich an den Mast binden, der Übergewichtige entfernt die Schokolade aus dem Haus-

2 Vgl. W. Mischel: The Marshmallow Test: Mastering Self-Control, 2014. 
halt, um der unmittelbaren Versuchung nicht nachgeben zu können. Manche unter uns trinken keinen Alkohol vor Sonnenuntergang oder haben wie Leonid Breshnew eine Zigarettendose, an deren Inhalt man nur jede Stunde einmal gelangen kann.

Wir sind sogar bereit, für Selbstbindungsmöglichkeiten zu zahlen. Gerade Arme scheinen das zu tun. In Entwicklungsländern etwa nehmen sie Kredite zu hohen Zinsen auf, um eine zukünftig benötigte Summe sogleich zu weit niedrigeren Zinsen bei einer Bank wieder fest anzulegen. Sie zahlen dafür, das potenzielle Opfer von Geldeintreibern zu werden, nur um das langfristige Ziel ungeachtet der eigenen Alltagsschwäche erreichen zu können. ${ }^{3}$ In der entwickelten Welt besteht das Problem natürlich ebenfalls und, wie überall, nicht nur für die Armen. Unter rechtlich entwickelten Institutionen werden sogar spezialisierte Selbstbindungsverträge angeboten. ${ }^{4}$

Falls Odysseus nach einem Mast fragt, an den er sich binden will, kann man vom Standpunkt individueller Entscheidungsautonomie zunächst nichts dagegen einwenden, wenn inm dazu auf freien Vertragsmärkten entsprechende Angebote gemacht werden. Falls es jedoch insoweit Marktversagen geben sollte und keine geeigneten Selbstbindungen angeboten werden, entstehen Versuchungen für den Staat, sich als Anbieter zu betätigen. Dies soll er bei Marktversagen, wenn es denn vorliegt. Eine ganz andere Sache ist es aber, wenn der Staat sich nicht nur als Mast-Bauer und Mast-Anbieter betätigt, sondern Odysseus dazu bringen oder gar verpflichten will, sich anzubinden. Genau darum geht es beim sogenannten „Nudging“. Man geht davon aus, dass keine hinreichenden Bindungsmöglichkeiten bestehen oder diese aufgrund menschlicher Schwäche nicht hinreichend genutzt werden. Als politische Gegenmaßnahme wird Odysseus nicht nur ein Mast geboten, sondern man bindet ihn mit leichten Fesseln, die es ihm freistellen, sich ohne größeren Aufwand entbinden zu lassen. Das geschieht zu seinen Gunsten und ohne sein vorheriges Einverständnis („Paternalismus"), aber mit der Option, sich von der Bindung zu befreien (,libertär").

\section{Ein gewichtiges Beispiel}

Man stelle sich einen Übergewichtigen vor, dem jemand den Eisschrank ausgeräumt hat, der sich aber jederzeit neu eindecken könnte. Es ist gut für inn, wenn er seinen Versuchungen nicht unmittelbar nachgeben kann. Weil er entscheidungsträge ist, macht er sich nicht auf, um den Eisschrank

3 Vgl. A. Banerjee, E. Duflo: Poor Economics: A Radical Rethinking of the Way to Fight Global Poverty, Reprint, New York 2012.

4 Vgl. I. Ayres: Carrots and Sticks: Unlock the Power of Incentives to Get Things Done, New York 2010. durch Einkäufe wieder zu befüllen, aber er könnte das jederzeit tun. Das klingt harmlos und ist es zunächst auch. Wenn etwa "die Kumpel“ den Eisschrank in dicker Freundschaft ausräumen, tun sie ihm einen Gefallen, dem er sich jederzeit entziehen kann. Er kann darauf beharren, dass der wohlgefüllte Eisschrank wieder zum Normalzustand (default) wird. Auf die Freunde ist in solchen Dingen nicht immer Verlass und der Staat könnte helfend eingreifen wollen, um den Übergewichtigen im Kampf mit den eigenen Pfunden beizustehen. Der Erlass einer „Eisschrankbefüllungsverordnung“ für Personen mit einem zu hohen Body-Mass-Index wäre „typisch“. Da es vorgelagerte Eingriffe in die Privatsphäre erfordern würde, würde man so etwas kaum akzeptieren wollen. Die Absurdität des Beispiels ist allerdings nicht zufällig. Sie weist darauf hin, dass akzeptables Nudging nicht nur die Austrittsoption zu geringen Kosten erfordert, sondern auch die Möglichkeit, den Anstoß (den Nudge) ohne massive Eingriffe in die Privat- und/oder öffentliche Sphäre zu erteilen. Das wird aber nur in Ausnahmefällen möglich sein.

In der Cafeteria der Schule zunächst die kalorienarmen und später und schwerer erreichbar die kalorienreichen Nahrungsmittel aufzubauen, ist eine Sache, alle Cafeterias dazu zu bringen, in dieser Weise zu verfahren, eine ganz andere. Wenn wir staatlich verlangen würden, dass alle Kaffeehäuser so vorgehen müssen, dann würden sie womöglich einfach keine kalorienarmen Produkte in ihr Programm nehmen wollen oder wir würden allen Kaffeehäusern verordnen müssen, kalorienarme Produkte in das Programm zu nehmen. Viele Drehungen der Interventions- und Regulierungsschraube drohen. Und wer „nudged dann die Nudger“?

\section{Gefährliches Nudging}

Bei näherer Betrachtung sind viele Phänomene, die sich im Zusammenhang mit dem Nudging stellen, überraschend kompliziert. Das deutet darauf hin, dass die Umsetzung des Konzeptes ungeachtet der Anfangsplausibilität vieler Beispiele problematisch ist. Sobald Nudging zum Programm der Politik gemacht wird, enthält es nicht mehr nur Ratschläge, sondern feste Regulierungen. Die einfache Aus- oder Rücktrittsoption allein kann dann jedenfalls nicht mehr für den libertären Charakter sorgen. Libertär bleibt zunächst jedes Nudging, das von Privaten gegenüber Privaten im Rahmen etablierter Rechte vorgenommen wird. Mehr Information darüber zu schaffen, wie wir uns besser selber managen und auf eigenen Wunsch nudgen lassen können, ist gewiss nicht zu kritisieren. Problematisch wird es, wenn man vorvertraglich oder „in contrahendo“, Nudges, die dann ja gerade nicht auf vor-vertragliche Zustimmung zurückgehen können, staatlich implementieren will. Das Argument, das man nicht vermeiden kann, dass in einer Entscheidungssituation eine Option als "default“ definiert sein muss und eintreten wird, wenn man nichts 
unternimmt, ist aber auch im Falle staatlich beeinflusster Entscheidungen triftig.

Wenn man die Erzwingung einer Entscheidung für legitim hält, dann ist es gewiss vernünftig, die „default option“ so zu gestalten, dass sie das Interesse des Entscheiders oder auch das anderer, die ein legitimes Interesse an der Sache haben, schützt. Der zuvor geübte Zwang ist zwar in keinem Falle libertär, doch wenn der Staat ohnehin Zwang in bestimmten Sphären ausüben muss - und das muss jeder annehmen, der nicht Anarchist ist - dann ist seine interessengemäße Gestaltung bestimmt wünschenswert. Der Zwang als solcher bleibt aber immer begründungsbedürftig. Die Austrittsoption und die Interessenausrichtung mildern das unvermeidliche Zwangsübel, doch dürfen wir uns von der Popularität des Nudgings nicht für die negative Seite des Zwangs desensibilisieren lassen. Betrachten wir die Sache noch etwas näher.

\section{Gefährliche Bindungen}

Wir erwarten von modernen Rechtsstaaten, dass sie Möglichkeiten rechtlicher Selbstbindung bereitstellen. Um die Erbringung dieser Leistung zu unterstützen, muss der Gesetzgeber ermächtigt werden, Regeln zu erlassen. Der ermächtigte Gesetzgeber kann aber unter dem Deckmantel, unsere freie Selbstbestimmung erweitern und uns vor deren selbstschädigendem Missbrauch schützen zu wollen, zum Instrument „paternalistischer" Interessenpolitik werden. Wir selbst wissen, dass mit der Größe der Bindungsmacht auch deren Risiken für uns zunehmen. Diese mit der Schattenseite des Nudgings verwandten Probleme muss man hinsichtlich der korrekten Bestimmung der staatlichen Rolle im Auge behalten.

\section{Doch prüfe, wer sich ewig bindet}

In unserem Recht gibt es spezielle Rücktrittsklauseln für den Haustür-Verkauf und andere für die Vertragsschließung im Internet. Der Bürger wird davor geschützt, sich übereilt selbst zu binden. Er kann sogar gegen sich selbst ein Verbot aussprechen, das inm untersagt, Spielbanken zu betreten. Der rechtliche Umgang mit dem Spiel ist auch sonst aufschlussreich. Denn die Rechtsordnung setzt Spielschulden generell nicht durch. Sie lässt es zudem nicht zu, dass es möglich ist, auf Kredit zu spielen (so dass der einzelne, der eine Spielbank betritt, mit einer zuvor abgezählten Geldsumme seine eigenen spontanen Wünsche partiell kontrollieren kann). Alle diese Regelungen haben einen (selbst-)paternalistischen Aspekt. Die sogenannte Vertragsfreiheit ist eine vom Staat definierte und beschränkte Ermächtigung zur Modifikation rechtlicher Ansprüche. Nicht alle Verträge werden vom Staat durchgesetzt, sondern nur bestimmte. Eine solche Beschränkung beinhaltet den Verzicht auf staatliche Durchsetzung von Klassen von Verträgen, nicht das Verbot entsprechender freiwilliger Transaktionen. Am Ende soll der Bürger selbst über seine
Bindungen entscheiden können. Er wird jedoch vor staatlicher Erzeugung von Dauerwirkungen in bestimmten Fällen geschützt. Auf Dauer angelegte Verträge, die keine Rücktrittsoder Austrittsklausel enthalten, sind wegen der Risiken, die sie erzeugen, problematisch. An sich könnte man sich vorstellen, dass der Staat anbietet, neben Eheverträgen mit auch solche ohne Scheidung durchzusetzen. Es bliebe den mündigen Bürgern dann selbst überlassen zu wählen, an welchen „Mast“ sie sich binden wollen. Doch sogar Wilhelm von Humboldt meinte, dass es einem früheren Selbst nicht erlaubt sein sollte, die späteren Manifestationen der gleichen Person in dieser endgültigen Weise an Neuorientierungen zu hindern. ${ }^{5}$ Dieses „paternalistische“ Element im Angebot von Selbstbindungsinstrumenten scheint unvermeidbar.

Die Rechtsordnung ist allerdings anscheinend weniger sensibel, was die automatische Verlängerung von Verträgen anbelangt. Einerseits möchte sie ebenso wie die Bürger, dass erwachsene Personen Sparverträge abschließen können, die sich automatisch verlängern. Der Bürger kann sich selbst angesichts seiner Neigung, lieber keine Entscheidung zu treffen, an die Fortführung seines Sparentschlusses auf milde Weise binden. Er sieht voraus, dass die Beträge vom Konto abgebucht werden und er voraussichtlich nicht die Entschlusskraft aufbringen wird, den Status quo vertraglicher Bindung zu ändern. Auf der anderen Seite verstehen etwa die Anbieter von Mobilfunkverträgen sehr genau, wie sie die entsprechenden psychologischen Mechanismen für sich nutzen können. Soll man Bürger nicht vor bestimmten, sich selbstverlängernden Verträgen schützen und innen andere Verträge nahelegen? Klar ist, dass es keine generelle verfassungsrechtliche Regel gegen sich selbst verlängernde Verträge geben darf, wenn man die guten Seiten solch weicher Bindungen etwa im Falle sich selbst verlängernder Sparverträge erhalten will. Man braucht sehr gute Gründe, warum der Staat gegen ähnliche Vertragsarten diskriminieren darf. Eine wesentliche Funktion der Privatrechtsordnung ist es schließlich, privaten Subjekten die Selbstbindung zu ermöglichen und die entsprechenden Bindungen dann mit Mitteln der staatlichen Rechtsdurchsetzung wirksam werden zu lassen. Aber eine Diskriminierung bestimmter und eine Förderung anderer Bindungen ist angesichts viel stärkerer staatlicher Eingriffe, die uns nun ebenfalls als Nudging nahegebracht werden sollen, vergleichsweise harmlos.

\section{Bindungsgebote und echte Nudges}

Als Beispiel für Nudges werden in Deutschland manchmal die sogenannten "gesetzlichen Versicherungen" genannt. Krankenpflichtversicherung und Rentenpflichtversicherung sind jedoch - ähnlich wie die Automobilhaftpflichtversicherung

5 W. von Humboldt: Ideen zu einem Versuch, die Grenzen der Wirksamkeit des Staats zu bestimmen, Stuttgart 1851/1967. 
gerade nicht im Sinne eines „Nudging“ zu verstehen. Wären sie bloße Nudges, dann müsste allen Menschen zugestanden werden, aus den Systemen gänzlich herauszuoptieren. Das ist nicht der Fall. Es handelt sich nicht um (selbst-)paternalistische Regelungen, sondern um Zwangseinrichtungen zur Abwehr von Externalitäten. So wird zwar die gesetzliche Krankenversicherung gern mit dem Argument vertreten, man müsse diejenigen, die nicht selbst für sich Krankenversicherungsvorsorge treffen können, weil sie zu uneinsichtig oder weil sie zu arm sind, zu ihrem eigenen langfristigen Vorteil unterstützen. Aber durch die Pflichtversicherung werden nicht primär die Zwangsversicherten geschützt, sondern vor allem die Gesellschaft. Wir würden einem Samariter-Dilemma unterliegen. ${ }^{6}$ Denn wir würden zahlen wollen, weil wir es rechtsstaatlich nicht aushalten, Bürger für ihre Fehler sterben zu lassen. Für zweckgebundene Gesundheitssteuern gibt es insoweit gute Gründe. Aber in ihnen Nudges sehen zu wollen, führt zu solchen Perversionen wie die Entbindung der Wohlhabenden von der Steuer und ihrer Umverteilungskomponente, die allein von den weniger Wohlhabenden zu tragen ist. Der Staat darf uns im Falle echten Nudgings gerade nicht der letzten Verfügungsmacht enteignen. Zwangsversicherungen tun aber genau das.

\section{Schlussüberlegung}

Es scheint ganz im Sinne von Franz Böhms ${ }^{7}$ ordnungspolitischer Vorstellung von einer Privatrechtsgesellschaft zu liegen, wenn der Staat beliebige Selbstbindungen durchsetzt,

6 J. M. Buchanan: The Samaritan's Dilemma, in: E. S. Phelps (Hrsg.) Altruism, Morality and Economic Theory, New York 1975, S. $77 \mathrm{ff}$

7 F. Böhm: Privatrechtsgesellschaft und Marktwirtschaft, in: ORDO, Bd. 17, 1966, S. 75-151. deren Wahl und Umfang aber den Bürgern selbst überlässt. Doch selbst Wilhelm von Humboldt hat in seinen „Ideen zu einem Versuch, die Grenzen der Wirksamkeit des Staats zu bestimmen"8 die wirksame Durchsetzung beliebiger Verträge durch den Staat wegen der inhärenten Risiken abgelehnt. Auch in seinem libertären Ansatz werden Bürger durchaus paternalistisch vor den Gefahren zu weitgehender rechtlicher Selbstbindungsmöglichkeiten durch Rücktritts- und Beschränkungsklauseln bewahrt. Es sollte genau verstanden sein, dass man im sogenannten „libertären Paternalismus“, den man heute mit Nudging verbindet, weiter als Humboldt gehen will. Die Entscheidungssituation, in der Individuen selbst über Selbstbindungen entscheiden, soll staatlich beeinflusst werden. Zwar geht es im Falle echten Nudgings nicht darum, dem Bürger etwas aufzuzwingen, sondern in vorvertraglichen Entscheidungssituationen, in denen er sich nach wie vor gegen bestimmte, nach allgemeinen Maßstäben wünschenswerte Aktionen entscheiden kann, die Wahl bestimmter Aktionen zu fördern. Vor allem wenn dieser eingeschränkte Eingriff auf verhaltenstheoretisch fundiertem „choice editing" beruht, hat er positive Seiten. Er bleibt aber problematischer als die Weigerung des Staates, bestimmte Vertragsbindungen zu ermöglichen. Das letztere, harmlosere Mittel sollte man zunächst einsetzen. Vor allem, wenn es darum geht, eine zunehmend alternde Bevölkerung davor zu schützen, in ihren eingefahrenen Vertragsbeziehungen zu verharren, könnte der Staat sich durchaus weigern, gewisse Arten von Verträgen gerichtlich durchsetzbar zu machen. Das Hauptproblem wird sein, dies nach allgemeinen Prinzipien zu vollziehen, die nicht zu altersdiskriminierender Interessenpolitik einladen.

8 W. von Humboldt, a.a.O.

\section{Steven Bosworth, Simon Bartke}

\section{Implikationen von Nudging für das Wohlergehen von Konsumenten}

Drei kürzlich ausgeschriebene Stellen am Bundeskanzleramt zeigen ein Interesse der Bundesregierung am Thema Nudging auf. Die Mitarbeiter dieser Stabsstelle sowie damit verbundene Entscheidungsträger sollten sich dessen bewusst sein, dass die Gestaltung erfolgreicher Nudges ein Verständnis der Funktionsweise menschlicher Entscheidungsfindung und deren Auswirkung auf das Wohlergehen erfordert. Dieser Artikel stellt daher Anomalien beim Treffen wirtschaftlicher Entscheidungen vor, auf die Nudging abzielen soll. Außerdem werden die möglichen Effekte von Nudges auf das Wohlergehen von Konsumenten systematisch analysiert und somit eine Orientierung für die Entwicklung angemessener Nudges geliefert.
Ein Nudge ist ein Eingriff, der menschliche Entscheidungen beeinflussen soll, ohne die Zahl möglicher Optionen einzuschränken. Das Konzept des Nudgings entstammt Erkenntnissen aus der Verhaltensökonomie, die aufzeigen, dass Menschen nicht immer die Entscheidungen treffen, die das Wohlergehen maximieren. Dies kann eintreten, weil Menschen nicht fähig sind, alle relevanten Aspekte in die Entscheidung miteinzubeziehen, sie unangemessene Erwartungen über die Konsequenzen ihrer Entscheidungen haben, oder weil es innen an Willenskraft fehlt, die bevorzugte Alternative tatsächlich umzusetzen. Weiterhin können Präferenzen vom Umfeld abhängig sein, in dem Entscheidungen getroffen werden. Diese Verzerrungen beim Entscheiden, im Weiteren 
„Anomalien“ (biases) genannt, wurden in Jahrzehnte andauernder verhaltensökonomischer Forschung entdeckt, getestet und modelliert. ${ }^{1}$ Unterschiedliche Arten von Anomalien erfordern unterschiedliche Nudges und haben unterschiedliche Implikationen für das Wohlergehen.

Eine Quelle von Anomalien in der Entscheidungsfindung sind psychische oder kognitive Kosten, die durch das Vergleichen von unterschiedlichen Entscheidungsalternativen miteinander entstehen. Diese Kosten können mit der Komplexität des Entscheidungsproblems zunehmen und als eine Art von Transaktionskosten angesehen werden. Andere Anomalien resultieren aus verzerrten Einstellungen. Die verhaltensökonomische Evidenz belegt die Tendenz von Menschen, die eigenen Fähigkeiten im Vergleich zu anderen zu optimistisch einzuschätzen. Menschen neigen außerdem dazu, Muster in voneinander völlig unabhängigen Ereignissen zu erkennen, in denen kein Muster bestehen kann. Die Präferenzen von Entscheidern können sich auch in vorhersagbarer Weise zwischen dem Moment der Entscheidung und dem Moment, in dem sich die Konsequenzen der Entscheidung offenbaren, verändern (jeder, der einmal eine Diät versucht hat, oder aufhören wollte zu rauchen, kann sich da sicherlich hineinversetzen). Zusätzlich beurteilen Menschen die Attraktivität von verschiedenen Entscheidungsalternativen, indem sie sie mit einem Referenzzustand vergleichen. Dieser Referenzzustand verändert sich jedoch auch im Laufe der Zeit in Abhängigkeit von den eigenen Umständen. Eine Erkenntnis aus der „Prospect Theory" ${ }^{2}$ ist, dass ein gegebener Verlust in Bezug auf diesen Referenzzustand einen stärkeren Einfluss auf die Attraktivität einer Entscheidungsoption besitzt als ein gleich hoher Gewinn.

Die Wünsche und Bedürfnisse von Menschen können vom Kontext, in dem sich diese zum Zeitpunkt der Entscheidung befinden, beeinflusst werden. Dies kann daran liegen, wie ein Entscheidungsumfeld wahrgenommen wird oder auch an der Interaktion zwischen dem Umfeld und dem affektiven Zustand des Entscheiders (z.B. eine positive Einstellung durch gutes Wetter). Subtile Veränderungen in der Präsentation der Entscheidungsoptionen können daher entscheidende Effekte auf die letztliche Entscheidung haben. Die Anfälligkeit menschlichen Entscheidungsverhaltens für solche Faktoren erhöht den Schwierigkeitsgrad, das Wohlergehen des Entscheiders nach einer derart beeinflussten Entscheidung zu beurteilen sowie zu bewerten, ob ein Nudge, der zu dieser Entscheidung geführt hat, die Wohlfahrt erhöht hat.

1 Vgl. M. Allais: Le comportement de l'homme rationnel devant le risque: critique des postulats et axiomes de l'école Americaine, in: Econometrica, 21. Jg. (1953), H. 4, S. 503-546; H. A. Simon: Models of man; social and rational, New York 1957, Kap. 14, S. 287 ff.; D. Kahnemann, A. Tversky: Prospect Theory: An analysis of decision under risk, in: Econometrica, 47. Jg. (1979), H. 2, S. 263-291.

2 Ebenda.

\section{Anomalien mit Hilfe von Nudges überwinden}

Nudges, die das Wohlergehen der Konsumenten maximieren, können Kosten oder zusätzliche Regulierungen für die Produzenten bedeuten. Diese können das Wohlergehen der Produzenten verschlechtern. Diese Thematik sollte von relevanten Entscheidungsträgern besonders wegen möglicher politökonomischer Auswirkungen beachtet werden, entzieht sich jedoch dem Rahmen dieses Artikels. Da die Entscheidungsanomalien der Handelnden zu Entscheidungen führen können, die diese nach genauerer Überlegung gerne anders getroffen hätten, können Maßnahmen von politischen Entscheidungsträgern genutzt werden, um diese Anomalien in ihren schädlichen Auswirkungen einzugrenzen. Diese Argumentation motivierte traditionelle paternalistische Maßnahmen wie Steuern auf Zigaretten, das Verbot von Betäubungsmitteln und Regulierungen zum Wucherzins. Die Art dieser Entscheidungsanomalien öffnet jedoch auch die Tür für „libertären Paternalismus“. Im Gegensatz zum reinen Paternalismus beeinflusst dieser Entscheidungen nicht dadurch, dass Entscheidungsmöglichkeiten verboten oder eingeschränkt werden, die Wahlfreiheit soll vollständig intakt bleiben. Traditionell werden wirtschaftliche Maßnahmen danach beurteilt, ob sie das Wohlergehen erhöhen, was bei Abwesenheit von Anomalien ein wohldefiniertes Kriterium ist. Klassischerweise sind Ökonomen der Auffassung, dass die Entscheidungen von Handelnden exakt aufzeigen, was diese Handelnden wollen. Daher können politische Maßnahmen in dieser Betrachtungsweise das Wohlergehen nur in den Situationen verbessern, in denen die Entscheidungen der einen Partei einer anderen Partei Schaden verursachen (Externalitäten).

Die Wohlfahrtsimplikationen, die sich aus Maßnahmen im Stile des libertären Paternalismus ergeben, zu bewerten, ist jedoch viel komplizierter. Thaler und Sunstein ${ }^{3}$ geben den normativen Idealfall eines Nudges wie folgt an: Ein „reiner“ Nudge befähigt Menschen dazu, eine Entscheidung zu treffen als seien sie frei von Entscheidungsanomalien. Unserer Meinung nach ist das eine gute theoretische Benchmark, wir stellen aber auch inhärente Schwierigkeiten in der Implementierung dieses Idealfalls fest. Die Wohlfahrtseffekte einer durch Nudging beeinflussten Entscheidung sollten auch klassisch danach beurteilt werden, ob sie Externalitäten wie Umweltverschmutzung reduzieren. Zusätzlich sind sie aber danach zu bewerten, ob die Ergebnisse von "genudgten" Entscheidungen letztlich mehr den tatsächlichen Präferenzen entsprechen, als wenn der mit Anomalien behaftete Entscheider ohne den Nudge entschieden hätte. Dies stellt ein schwieriges Unterfangen dar, da die Anomalien die Übereinstimmung zwischen Zielen und Präferenzen verzerren und somit zunächst alles diagnostiziert

3 Vgl. R. H. Thaler, C. R. Sunstein: Nudge: Improving decisions about health, wealth, and happiness, New Haven, CT, 2008. 
werden muss: Entscheidungsanomalien mit ihren negativen Effekten auf das Wohlergehen der Konsumenten sowie daraus resultierende Externalitäten. Zusätzlich zu diesem Identifikationsproblem entstehen jedoch auch normative Fragen für Entscheidungsträger. Welche Präferenzen der Konsumenten sollen als die tatsächlichen Präferenzen angesehen werden? Welche Nudges sollen diese Präferenzen in welche Richtung beeinflussen?

\section{Voreingestellte Entscheidungen}

Zunächst untersuchen wir die Nudges, die auf Entscheidungsanomalien abzielen, die im Zusammenhang mit den Kosten der Entscheidungsfindung auftreten. Als Beispiel sei das Entscheidungsproblem, sich für oder gegen den Status eines Organspenders zu entscheiden, betrachtet. Die Kosten, die bei der Entscheidungsfindung entstehen, nennen wir c. Diese Kosten können von Person zu Person variieren. Nehmen wir an, dass in der Abwesenheit einer aktiven Entscheidung die Option „Nicht-Spender" automatisch für jede Person ausgewählt wird. In diesem Fall bekommen die, die einen Status als Spender für sich zwar bevorzugen würden, aber nicht so sehr, dass sie bereit wären c einzugehen, automatisch keinen Spenderstatus. Diejenigen, die es bevorzugen, kein Spender zu sein, müssen jedoch kein c tragen. Personen, die es stark - zu einem Grad größer als c - bevorzugen, Spender zu sein, werden weiterhin den Status als Spender wählen. Falls nun die voreingestellte Entscheidung (Default) auf den Status als Organspender gesetzt wird, dann verbessert sich das Wohlergehen derjenigen, die den Status als Organspender nur leicht bevorzugen, also zu einem Grad weniger als c. Dieser Wohlfahrtsgewinn wird nicht die Kosten übersteigen, die Entscheidung für die Organspende selbstständig zu treffen, da sie diese Entscheidung sonst getroffen und somit starke Präferenzen für die Organspende gezeigt hätten. Demgegenüber wird durch das Umstellen der voreingestellten Entscheidung aber potenziell das Wohlergehen derjenigen verschlechtert, die leichte Vorlieben dafür haben, kein Spender zu sein. Falls deren Zahl und Entscheidungskosten vergleichbar mit denen sind, die für sich den Status des Spenders leicht bevorzugen, kann der Wechsel der voreingestellten Entscheidung auf den Status „Organspender" jeglichen Wohlfahrtsgewinn zunichtemachen. Hier wird deutlich, dass Nudges, die die voreingestellte Entscheidung verändern, nur dann das Wohlergehen erhöhen, wenn die Entscheidungskosten hoch sind und Präferenzen annähernd homogen sind. Im Rahmen dieses Nudges sollten Entscheidungsträger daher auf dieses Kriterium achten. Ein bekanntes Beispiel ist hier die automatische Teilnahme an einem Renten-Sparplan in den USA. ${ }^{4}$ Im Falle von Vorsorgeent-

4 Vgl. B. C. Madrian, D. F. Shea: The Power of Suggestion: Inertia in 401(k) Participation and Savings Behavior, in: Quarterly Journal of Economics, 116. Jg. (2001), H. 4, S. 1149-1187. scheidungen fürs Alter scheinen recht homogene Anomalien in der Entscheidungsfindung vorzuliegen. Im Zusammenhang mit der Entscheidung für Finanzprodukte werden auch Anomalien durch Entscheidungskosten deutlich: Wer hat Zeit und Muße, das ganze Kleingedruckte detailliert zu verstehen und vergleichen zu können?

\section{Aktive Wahl}

Derselben Logik folgt die Analyse anderer Nudges, die Entscheidungskosten adressieren könnten. Betrachtet wird eine Maßnahme, die für jeden verpflichtend eine aktive Wahl vorsieht, sodass keine voreingestellte Entscheidung gesetzt werden muss. Durch diese Maßnahme wird sichergestellt, dass jeder, der kein Organspender sein will, auch keiner wird, sowie jeder, der Organspender sein will, Spender wird. Diese Maßnahme scheint auch politstrategisch attraktiv, da der Staat nicht seine Auffassung von der voreingestellten Entscheidung durchsetzen muss. Hier ist jedoch zu beachten, dass nur Gruppen mit leichten Vorlieben am Ende mit einer für sie ungünstigen voreingestellten Entscheidung belassen werden (siehe oben). Diese Gruppen wollten c nicht tragen und haben daher keine Entscheidung getroffen. Eine Maßnahme, durch die jeder eine Wahl treffen muss, ist dann eindeutig wohlfahrtsreduzierend, wenn man davon ausgeht, dass Menschen ihre Vorlieben für den Status als Spender oder Nicht-Spender korrekt ausdrücken.

Maßnahmen, die die Kosten (bei) der Entscheidungsfindung reduzieren sollen, sind beispielsweise die deutliche und verständliche Darstellung von relevanten Produkteigenschaften am Produkt oder der Abbau bürokratischer Hürden. Dadurch werden Konsumenten mit schwachen Vorlieben gegenüber einer voreingestellten Option eher ihre Präferenzen durch eine aktive Entscheidung ausdrücken und ihr Wohlergehen wird sich verbessern. Gleichzeitig muss niemand, der die voreingestellte Option bevorzugt, höhere Kosten tragen. Diese Maßnahme erhöht eindeutig das Wohlergehen, und Entscheidungsträger müssen hier auch nicht die wahre Verteilung von Vorlieben kennen.

Verzerrte Vorstellungen über die Konsequenzen einer Entscheidung stellen hingegen ein relativ einfaches Problem für Entscheidungsträger, die über den Einsatz von Nudges nachdenken, dar. Nehmen wir beispielsweise an, Haushalte seien der Überzeugung, dass herkömmliche Glühlampen über deren gesamte Lebensdauer kostengünstiger sind als effiziente LED-Lampen. Diese Meinung kann entstehen, falls man die jeweiligen Kosten des Energieverbrauchs über die Lebensdauer nicht ausreichend berücksichtigt und zu viel Gewicht auf den anfänglichen Anschaffungspreis legt. Wenn man davon ausgeht, dass das Wohlergehen von den tatsächlichen und nicht den erwarteten Kosten abhängt, kann es das Wohlergehen der Konsumenten erhöhen, wenn man sie deutlich 
und verständlich über die Kosten der gesamten Lebensdauer beider Optionen aufklärt. Dies würde die Entscheidung der Konsumenten besser mit den letztlich erfahrenen Kosten in Einklang bringen. Diejenigen Konsumenten, die tatsächlich herkömmliche Glühlampen bevorzugen - vielleicht wegen der Farbe des Lichts - werden von diesen Informationen nicht beeinflusst sein, so lange die Präferenz der Lichtfarbe die wahren Kosten der Lampe kompensiert.

Das Umfeld und die Präsentation eines Entscheidungsproblems können einen starken Einfluss auf die getroffene Entscheidung ausgeübt haben. Da Menschen Gewinne und Verluste unterschiedlich wahrnehmen und bewerten, können Nudges eingesetzt werden, die Entscheidungen entweder in einem Gewinn- oder Verlustkontext präsentieren (Framing). Im Glühlampenbeispiel können die unterschiedlichen Energiekosten von klassischen Glühlampen und LED-Lampen entweder aufgezeigt werden als: a. nicht auf LED-Lampen zu wechseln kostet Sie 50 Euro jedes Jahr oder b. durch den Wechsel auf LED-Lampen sparen Sie 50 Euro jedes Jahr. Da Menschen sensibler auf Verluste reagieren, sollte die erste Darstellung gewählt werden. Die Wohlfahrtseffekte einer solchen veränderten Darstellung zu analysieren ist nicht trivial, da Präferenzen vom Entscheidungsumfeld abhängen. Entscheidungsträger müssen sich hier festlegen, ob sie die Vor-Nudge-Präferenzen von Konsumenten respektieren, bei denen diese mit den bisherigen Lampen zufrieden waren. Falls man jedoch nur Nach-Nudge-Präferenzen als relevant ansieht, dann erhöht dieser Nudge das Wohlergehen, da der Konsument nicht zu den ineffizienten Lampen zurückkehren wird.

Ein wichtiges empirisches Problem ist es im Rahmen des Nudgings zunächst auch, verzerrende Anomalien, die weite Teile der Bevölkerung betreffen und Konsumentscheidungen beeinflussen, zu identifizieren. Erst dadurch kann ein Nudging zur Beeinflussung dieser Anomalien überhaupt erst gerechtfertigt werden. In empirischen Studien bauen beispielsweise Hausmann sowie Dubin und McFadden ihre Identifikationsstrategien von Anomalien darauf auf, dass sie relative Marktanteile von unterschiedlichen Gütern vergleichen. ${ }^{5}$ Eine aktuelle Zusammenfassung findet sich bei Allcott. ${ }^{6}$

\section{Gesellschaftliche Normen}

Wenn man einen Nudge einsetzt, der das Entscheidungsumfeld beeinflussen soll, kann dies mit einem weiteren Ansatz

5 Vgl. J. Hausmann: Individual Discount Rates and the Purchase and Utilization of Energy-Using Durables, in: Bell Journal of Economics, 10. Jg. (1979), H. 1, S. 33-54; vgl. J. Dubin, D. McFadden: An Econometric Analysis of Residential Electric Appliance Holdings and Consumption, in: Econometrica, 52. Jg. (1984), H. 2, S. 345-362.

6 Vgl. H. Allcott: Paternalism and Energy Efficiency: An Overview, NBER Working Papers, Nr. 20363, National Bureau of Economic Research, Cambridge MA 2014. verknüpft werden: der Tendenz, gesellschaftliche Normen zu befolgen. Ein Beispiel dazu sind US-Studien über den Inhalt von Elektrizitätsrechnungen.? Die Rechnungen, die im Zuge der Experimentteilnahme an Haushalte versendet wurden, gaben Informationen darüber, wie hoch ihr eigener Elektrizitätsverbrauch im Vergleich zu dem ihrer Nachbarn ist. Diese Information wurde zusätzlich mit Emoticons versehen, die ein fröhliches Gesicht zeigten, falls der jeweilige Haushalt weniger Energie verbrauchte als der Nachbarschaftsdurchschnitt. Dieser Nudge erwies sich als effektiv, Haushalte dazu zu bewegen, sich mit ihrem Verbrauch dem Durchschnittsverbrauch anzunähern. Sie bewirkte auch eine Verringerung des gesamten Energieverbrauchs.

Angenommen, Menschen haben konsistente Präferenzen, gesellschaftlichen Normen zu folgen, dann bewegt der Nudge des Informierens über den Durchschnittsenergieverbrauch des Umfelds dazu, den eigenen Verbrauch in Richtung der tatsächlichen Präferenzen zu verschieben. Dieser Nudge kann auch die Einstellung der Menschen gegenüber ihrem Energiekonsum verändern. Hatten sie vorher bei ihrem Energiekonsum nur ihre persönlichen Bedürfnisse und Kosten im Auge, könnte sich dies dahingehend verändern, dass sie nun denken, ihr Konsum hat normative Bedeutung. Ohne den Vergleich mit dem Umfeld zieht der Haushalt Nutzen aus dem Energiekonsum; mit dem Vergleich könnte der Haushalt zusätzlich Nutzen daraus ziehen „das Richtige zu tun“. Eine Analyse des Wohlergehens unter diesen beiden Möglichkeiten ist nicht einfach, da diese beiden Arten des Nutzens einen unterschiedlichen Ursprung haben. Solche Nudges können ungewisse Auswirkungen auf das Wohlergehen haben. Sollten dadurch jedoch schwerwiegende negative Externalitäten wie Umweltverschmutzung vermieden werden, ist dies vielleicht stärker zu gewichten.

\section{Leitfaden für Konzeption und Einsatz von Nudges}

Der Leitfaden besteht aus folgenden zu klärenden Fragen:

1. Was ist das Problem, das gelöst werden soll? Welche Entscheidungsanomalie liegt vor und macht es möglich, dass ein Nudge das Wohlergehen verbessern kann?

2. Was sind die wahren Wünsche und Bedürfnisse der Menschen, die genudged werden sollen? Wie homogen oder inhomogen sind diese zwischen den Einzelnen?

3. Führt der Nudge dazu, dass sich die Wünsche und Entscheidungen der Individuen einander annähern? Wollen die Betroffenen genudged werden?

Die Antworten auf diese Fragen sind keinesfalls trivial, bieten aber einen guten Leitfaden für zukünftige Nudger.

7 Vgl. D. L. Costa, M. E. Kahn: Energy Conservation „Nudges“ And Environmentalist Ideology: Evidence From A Randomized Residential Electricity Field Experiment, in: Journal of the European Economic Association, 11. Jg. (2013), H. 3, S. 680-702; H. Allcott : Social Norms and Energy Conservation, in: Journal of Public Economics, 95. Jg. (2011), H. 9-10, S. 1082-1095. 


\section{Unvollständige Rationalität ist keine hinreichende Begründung für paternalistisches Eingreifen}

In den vergangenen Jahren hat sich ein blühender Zweig der verhaltensökonomischen Forschung mit der Frage beschäftigt, welche normativen Schlussfolgerungen aus den empirisch, oft experimentell nachgewiesenen Abweichungen menschlicher Entscheidungen vom ökonomischen Verhaltensmodell zu ziehen sind. . Die Mehrheit der einschlägigen Beiträge unterstellt, wenn auch oft nur implizit, dass die als deskriptives Modell gerade erst gescheiterte Vorstellung vollständig rationalen Verhaltens nun als normativer Maßstab dienen soll. ${ }^{2}$ Soweit man also empirisch beobachtet, dass Individuen sich systematisch und vorhersagbar anders verhalten als es ein relativ strikt und traditionell definierter Homo oeconomicus täte, hält man sie für ökonomisch therapiebedürftig.

Eine strikte Definition bedeutet hier vor allem, dass die Individuen durch eine widerspruchsfreie Präferenzordnung charakterisiert werden können und zeitkonsistent handeln. Sie sollen darüber hinaus gut informierte Entscheidungen treffen und keine systematischen Fehleinschätzungen der Restriktionen ihrer Entscheidungssituation tätigen. Letztendlich ist der Rationalitätsanspruch, an dem die Realität gemessen wird, also sehr nah am Modell vollständiger Rationalität, wie man es aus den Lehrbüchern der Mikroökonomik kennt. Dieser Anspruch ist hoch, und es ist nicht sehr überraschend, dass die Realität insbesondere bei kognitiv anspruchsvollen Entscheidungen unter Risiko und bei solchen, die längere Zeiträume betreffen, dem oft nicht entspricht.

Wenn man nun diskutiert, ob aus dem deskriptiven Scheitern einer bestimmten Rationalitätsannahme eine Notwendigkeit zum wirtschaftspolitischen Eingreifen abgeleitet werden kann, so ist zunächst zu bedenken, dass wir es hier in der Regel nicht mit negativen Externalitäten zu tun haben. Die betroffenen Individuen mögen für sich selbst suboptimale Entscheidungen treffen, aber eine direkte Schädigung anderer ist in aller Regel nicht festzustellen. Selbst eine indirekte Schädigung, beispielsweise in Form von höheren Kosten in den Sozialsystemen als Folge individuell ungesunder Konsumentscheidungen, ist empirisch äußerst umstritten

1 Vgl. für einen neueren Überblick etwa C. Sunstein: Behavioral Economics and Paternalism, in: Yale Law Journal, 122. Jg. (2013), S. 1826-1899.

2 Dies ist allerdings keine neue Diskussion. Vielmehr wird die Frage, ob Ökonomen ihre verschiedenen Modelle rationalen Verhaltens deskriptiv oder normativ verstehen, bereits seit den frühen Diskussionen um den axiomatischen Ansatz von John von Neumann und Oskar Morgenstern debattiert, vgl. F. Heukelom: Behavioral Economics: A History, Cambridge 2014. und für Beispiele wie das Rauchen ist sogar ein positiver monetärer Gesamteffekt für die Gesellschaft denkbar. ${ }^{3}$

Solange man aber eine erhebliche Schädigung Dritter nicht als Begründung für eine Intervention in individuelle Entscheidungen heranziehen kann, spricht einiges dafür, solche Eingriffe nur dann durchzuführen, wenn eine Nachfrage nach ihnen besteht. Dies ist jedenfalls dann das relevante Kriterium, wenn man davon ausgeht, dass die Legitimation politischer Eingriffe in den verallgemeinerbaren Interessen der Bürger zu suchen ist. ${ }^{4}$ Kann man also davon ausgehen, dass informierte Bürger es akzeptabel finden, durch paternalistische Eingriffe in ihren individuellen Entscheidungsprozessen gesteuert zu werden?

\section{Die verschiedenen Arten des Nudgings}

Es gibt einige relativ unproblematische Varianten der Hilfestellung zur besseren individuellen Entscheidungsfindung. Ein Beispiel ist das von R. Thaler und S. Benartzi konzipierte Programm mit dem Namen „Save More Tomorrow“. ${ }^{5}$ Hier geht es darum, das Problem des sogenannten hyperbolischen Diskontierens abzumildern, bei dem die Betroffenen durch eine zeitinkonsistente Präferenzordnung gekennzeichnet sind und Entscheidungen immer wieder verschieben, die mit kurzfristigen Kosten und langfristigem Nutzen verbunden sind. ${ }^{6}$

Der Lösungsansatz, den Thaler und Benartzi vorschlagen, ist so einfach wie transparent. Er besteht darin, dass die Individuen sich in einem (sogar jederzeit kündbaren) Vertrag selbst verpflichten können, einen Anteil zukünftiger Gehaltserhöhungen automatisch für ihre Altersvorsorge zu verwenden. Durch diese Selbstbindung wird zumindest bei einem Teil der Teilnehmer und zumindest für einen Übergangszeitraum die Sparquote erhöht und das Problem des Aufschiebens der Altersvorsorge bekämpft. Die Teilnehmer des Pro-

3 Vgl. etwa K. Viscusi: The Governmental Composition of the Insurance Cost of Smoking, in: Journal of Law and Economics, 42. Jg. (1999), S. 575-609.

4 Vgl. V. Vanberg: Konsumentensouveränität und Bürgersouveränität: Steuerungsideale für Markt und Politik, in: R. Czada, R. Zintl (Hrsg.): Politik und Macht, Politische Vierteljahresschrift, Sonderheft 34 , Wiesbaden 2003, S. 48-65.

5 Vgl. R. Thaler, S. Benartzi: Save More Tomorrow: Using Behavioral Economics to Increase Employee Saving, in: Journal of Political Economy, 112. Jg. (2004), S. S164-S187.

6 Vgl. D. Laibson: Golden Eggs and Hyperbolic Discounting, in: Quarterly Journal of Economics, 112. Jg. (1997), S. 443-477. 
gramms sind jederzeit über ihre Handlungsoptionen im Bilde und haben stets die Möglichkeit, selbst aktiv zu werden und die Konditionen des Programms zu ändern oder ganz auszutreten. Insofern kann man argumentieren, dass hier schlicht ihre Konsumentensouveränität erhöht und ihnen ein zusätzliches Instrument zur Planung inres Konsumpfades in der Zeit an die Hand gegeben wird. Dies trifft aber bei weitem nicht für alle Varianten des Nudgings zu - im Gegenteil.

Sehr häufig schlagen die Vertreter des neuen Paternalismus vor, sogenannte Framing-Effekte bewusst zu nutzen. ${ }^{7}$ Diese liegen dann vor, wenn durch das gezielte Gestalten einer Entscheidungssituation die Wahrscheinlichkeit, dass die Individuen sich für eine bestimmte Alternative entscheiden, systematisch beeinflusst werden kann. Solches Framing wirkt aber sehr oft nur dann, wenn die Betroffenen nicht wissen, dass es stattfindet. Ein klassisches Beispiel ist der Vorschlag, Lebensmittel in einer Kantine so anzuordnen, dass unentschlossene Konsumenten zum Gemüse gesteuert werden, während die Unverbesserlichen mit einer starken Präferenz für Schokoriegel ihre Droge in versteckten Regalen suchen müssen, sie aber letztendlich doch finden können. Macht man dieses Framing transparent, und hängt am Eingang ein Schild auf, das auf versteckte Schokoriegel hinweist, dann wird der verbleibende Effekt auf Dauer nicht besonders groß sein.

Ein anderes, vielleicht ernsthafteres Beispiel ist der Versuch, nicht über offene Information und Anreize, sondern über Ansprachen, die an emotionale Affekte appellieren, die Zahl der Organspender zu erhöhen, wie es das Behavioral-InsightsTeam der britischen Regierung vorschlägt. ${ }^{8}$ Hier wird deutlich, wie selbst in sensibelsten Fragen, die eigentlich eine sorgfältige Abwägung von Argumenten durch jeden Einzelnen erfordern, für Regierungen die Versuchung zum Einsatz manipulativer Instrumente zur Durchsetzung politischer Ziele besteht.

Es gilt also genau zu unterscheiden zwischen Instrumenten, die es den Individuen selbst erlauben, von innen erkannte Mängel ihrer Entscheidungsprozesse zu beheben und andererseits einem im engeren Sinne paternalistischen Ansatz, der die Individuen lenkt, ohne dass sie sich dieser Tatsache bewusst werden. Der neue Paternalismus hat daher im Vergleich zum traditionellen Paternalismus oft noch den Nachteil, dass die Betroffenen aufgrund des subtilen Framings überhaupt nicht wissen, dass sie beeinflusst werden und dann auch keinen Standpunkt zu diesem Instrumentarium einnehmen.

7 Vgl. etwa A. Tversky, D. Kahneman: The Framing of Decisions and the Psychology of Choice, in: Science, 211. Jg. (1981), S. 453-458.

8 Vgl. J. Schnellenbach: Neuer Paternalismus und individuelle Rationalität: eine ordnungsökonomische Perspektive, in: List-Forum für Wirtschafts- und Finanzpolitik, Nr. 40 (2014), S. 239-257.
Eine Sündensteuer auf Alkohol wird wahrgenommen und löst vielleicht auch politische Debatten aus, eine unauffällige Manipulation einer Entscheidungssituation bleibt dagegen oft unbemerkt und wird auch nicht zum Gegenstand von Diskussionen. Deshalb geht auch das Argument fehl, der neue Paternalismus lasse den Individuen die Freiheit, sich anders zu entscheiden, als der Paternalist es eigentlich anstrebt. Diese Freiheit setzt das Wissen um die Manipulation der Entscheidungssituation voraus, aber dieses Wissen fehlt in der Regel.

\section{Woher kommt der Rationalitätsüberschuss?}

Zwei notwendige Bedingungen für positive Wohlfahrtseffekte des neuen Paternalismus bestehen darin, dass externe Interventionen in individuelle Entscheidungsprozesse 1. unter realistischen Annahmen über die Verteilung von relevanten Informationen systematisch bessere Entscheidungen möglich machen und 2. diese Möglichkeit auch tatsächlich in effizienter Weise genutzt wird.

Schon im Hinblick auf die erste Bedingung sind Zweifel angebracht. Wie oben gesehen, kann man das Nicht-Abweichen der Individuen vom paternalistisch induzierten Verhaltensmuster nicht als Zustimmung deuten, sondern nur als erfolgreiche Manipulation. Damit er tatsächlich im Sinne der Betroffenen handelt, muss der Paternalist daher zuverlässige Informationen über ihre tatsächliche Präferenzordnung besitzen. Diese ist allerdings schwierig zu generieren: Die empirische Evidenz der verhaltensökonomischen Forschung zeigt ja gerade, dass die Voraussetzungen der Theorie offenbarter Präferenzen nicht gelten. Man kann also von beobachtetem Verhalten nicht eindeutig auf eine Präferenzordnung schließen. Vielmehr steht man vor einem Puzzle, das auf unterschiedliche Arten zu verschiedenen vollständigen Bildern zusammengesetzt werden kann.

Dies bedeutet aber, dass in der Praxis nicht so sehr die Orientierung an den wahren individuellen Präferenzen im Mittelpunkt stehen wird, sondern die Orientierung an den Werturteilen des Paternalisten selbst. Seine Präferenzen für sich und andere fließen in erheblichem Maß in die versuchte Rekonstruktion der Präferenzen seiner Mündel ein. So ist es auch nicht überraschend, dass bisher praktische Ansätze des neuen Paternalismus sich vor allem an plakativen - um nicht zu sagen: platten - gesellschaftlichen Konventionen orientieren und sich für die Identifizierung der wahren Interessen der Betroffenen kaum interessieren. ${ }^{9}$ Ein breit eingesetzter neuer Paternalismus würde dann vor allem dazu führen, solche Konventionen („Iss gesund!“, „Treibe mehr

9 Vgl. J. Schnellenbach: Nudges and Norms: The Political Economy of Soft Paternalism, in: European Journal of Political Economy, 28. Jg. (2012), S. 266-277. 
Sport!“, „Spare mehr!“ usw.) zu stabilisieren, abweichendes Verhalten noch mehr zu stigmatisieren und die langfristige Offenheit gesellschaftlicher Entwicklungen zu reduzieren. Ein systematischer, positiver Einfluss auf die individuelle Wohlfahrt ist dagegen nicht ersichtlich.

Selbst wenn die paternalistischen Planer über die wahren Präferenzen der Individuen gut informiert wären, ist dies noch keine Garantie dafür, dass das paternalistische Instrumentarium sinnvoll eingesetzt wird. Tatsächlich sind viele der Probleme, die durch eine paternalistische Politik korrigiert werden sollen, in politischen Entscheidungsprozessen vermutlich noch virulenter als in privaten Entscheidungen. Beispiele dafür sind die extreme Kurzfristorientierung ${ }^{10}$ oder auch die Neigung, aus aktuellen Ereignissen vollkommen falsche Risikoeinschätzungen zu extrapolieren. ${ }^{11}$ Ebenso ist es nicht unwahrscheinlich, dass verteilungspolitische und interessengruppenspezifische Motive auch hier eine erhebliche Rolle spielen.

Dies alles ist aber im Zusammenhang mit dem neuen Paternalismus problematischer als bei politischen Instrumenten, weil dieser wie oben gesehen oft auf ein intransparentes Vorgehen setzt, um erfolgreich zu sein. Wenn ein BehavioralInsights-Team die Exekutive berät, in welcher Weise sie ihre Ziele besonders effektiv durchsetzen kann, dann bedeutet dies oft nichts anderes, als dass die Politik auf Manipulation anstelle von Überzeugungskraft und rationalen Argumenten setzt. Dies ist zwar sicherlich nicht das, was akademische Befürworter des neuen Paternalismus anstreben; es ist aber das, was unter Berücksichtigung polit-ökonomischer Einflüsse zu erwarten ist.12 In dieser Hinsicht erleben wir auf dem Gebiet der Verhaltensökonomik eine Neuauflage der Diskussion zwischen Public Economics und Public Choice, wobei die eine Seite Vorschläge für eine effiziente Politik unter idealen politischen Bedingungen macht und die andere Seite darauf hinweist, dass eine solche Politik kein Resultat realer politischer Prozesse sein kann und dass der Versuch ihrer Implementierung sogar schädliche Effekte hervorbringt.

\section{Es geht auch ohne neuen Paternalismus}

Die Vertreter des neuen Paternalismus haben bisher eigentlich kein überzeugendes Argument dafür geliefert, dass vollständige individuelle Rationalität als normativer Maßstab und Ziel politischer Interventionen dienen soll. Dies ist umso relevanter, wenn man bedenkt, dass Märkte auch dann gut funktionieren (in dem Sinne, dass sie einen effizienten Allo- kationsmechanismus bereitstellen), wenn die individuellen Präferenzen der Marktteilnehmer nicht die traditionellen Rationalitätsannahmen erfüllen. ${ }^{13}$

Möglicherweise wird, indem über paternalistische Interventionen diskutiert wird, der Lösungsansatz für Probleme individueller Entscheidungen schlicht auf der falschen Ebene gesucht. Es ist beispielsweise nicht zu leugnen, dass die meisten Menschen - manche mehr, manche weniger - mit irgendeiner Form von Selbstkontrollproblemen zu kämpfen haben und sich auf dem einen oder anderen Gebiet so verhalten, dass ihre Entscheidungen mit einem Modell zeitinkonsistenter Präferenzen gut beschrieben werden können. Aber ist es nicht zu erwarten, dass die betroffenen Individuen selbst nach Lösungen für solche Probleme suchen, sofern sie überzeugt sind, dass sie tatsächlich ein relevantes Problem haben?

Auf dieser Ebene kann die verhaltensökonomische Literatur dann tatsächlich auch gute Ratschläge geben. Sie kann die Individuen anleiten, sich eigene Mechanismen zur Selbstbindung zu schaffen, und sie kann sie darüber aufklären, in welche Entscheidungsfallen Menschen häufig hineinlaufen. Nicht zuletzt können auch privatwirtschaftliche, maßgeschneiderte und individualisierte Angebote zur Korrektur von wirklich relevanten Entscheidungsverzerrungen gemacht werden. Das oben erwähnte Programm „Save More Tomorrow" wurde von Richard Thaler und Shlomo Benartzi als Handelsmarke registriert und vermarktet. Der in Yale lehrende Ökonom lan Ayres bietet eine kommerzielle Internetplattform zur Selbstbindung bei zeitinkonsistentem Verhalten an, ${ }^{14}$ und dies sind nur zwei Beispiele.

Wenn nun die Nachfrage nach solchen Mechanismen zwar vorhanden, aber nicht spektakulär groß ist, so könnte man dies auch als Hinweis darauf lesen, dass die Menschen sich überhaupt nicht in großem Maße für verhaltensökonomisch therapiebedürftig halten und mit ihren kleinen Abweichungen vom Ideal vollständiger Rationalität insgesamt gut zurechtkommen. Interessant ist hier erste empirische Evidenz, die keinen Zusammenhang zwischen dem Niveau individueller Selbstkontrolle und der Nachfrage nach neuem Paternalismus findet. ${ }^{15}$ Allerdings findet sich ein positiver Zusammenhang zwischen dem eigenen Grad der Selbstkontrolle und der Befürwortung von „hartem“ Paternalismus wie Verboten und Sündensteuern: Wer sich selbst unter Kontrolle hat, möchte scheinbar auch die anderen unter Kontrolle halten.

13 Vgl. R. Sugden: The Opportunity Criterion: Consumer Sovereignty without Coherent Preferences, in: American Economic Review, 94. Jg. (2004), S. 1014-1033.

14 Vgl. http://www.stickK.com/.

15 Vgl. S. Kragh Pedersen, A. K. Koch, J. Nafziger: Who Wants Paternalism?, erscheint in: Bulletin of Economic Research, 2014. cago Law Review, 73. Jg. (2006), S. 133-156.

11 Vgl. T. Kuran, C. Sunstein: Availability Cascades and Risk Regulation, in: Stanford Law Review, 51. Jg. (1999), S. 683-768.

12 Siehe auch C. Schubert, J. Schnellenbach: Behavioral Political Economy: A Survey, CESifo Working Paper, Nr. 4988, München 2014. 
Fazit

Etwas provokant überspitzt könnte man also sagen: Der neue Paternalismus versucht ein Problem zu lösen, das es gar nicht gibt, scheitert aber an der Lösung und öffnet stattdessen die Arena für ganz neue, polit-ökonomische Schwierigkeiten. Dieser Beitrag streitet ausdrücklich nicht ab, dass es auf der individuellen Ebene systematische Verzerrungen und Fehler in Entscheidungsprozessen gibt. Diese Phänomene scheinen aber von den Individuen selbst nicht als so gravierend betrachtet zu werden, dass sie Hilfe nachfragen würden. Für die fehlende Nachfra- ge gibt es gute Gründe: Der neue Paternalismus ist unter realistischen Annahmen kein effektives Instrument zur Verbesserung individueller Entscheidungen, sondern stochert im Hinblick auf die wahren Interessen der Betroffenen im Nebel und birgt erhebliche Gefahren der intransparenten Manipulation in sich. Insofern kann man argumentieren, dass, wenn schon interveniert wird, aus einer polit-ökonomischen Sicht der klassische, „harte“ Paternalismus noch zu bevorzugen wäre: Dieser ist immerhin transparent und veranlasst den Bürger, sich über seine individuelle Einstellung zur paternalistischen Steuerung Gedanken zu machen.

Joachim Weimann

\section{Nudging: Missbrauch der Verhaltensökonomie oder sinnvolle Politikberatung?}

Spätestens seitdem Bundeskanzlerin Merkel eine Stelle für Verhaltensökonomen ausgeschrieben hat, ist „Nudging“ auch in der deutschen Öffentlichkeit angekommen. Woher kommt dieses Interesse der Politik an den Erkenntnissen der Verhaltensökonomen? Sollen sich Verhaltensökonomen darüber freuen, oder sollten sie lieber Distanz wahren und zur Vorsicht mahnen? Und schließlich: Wie stellt sich die Sache für die Bürger unseres Landes dar, die „geschubst“ werden sollen?

\section{Dienstleister der Politik}

Wirtschafts- und Sozialwissenschaftler werden von Politikern sehr häufig als eine Art Dienstleister verstanden, deren Aufgabe es ist, die Politik darin zu beraten, wie sich politisch definierte Ziele und Absichten am besten durchsetzen lassen. Etwas verharmlosend ist in diesem Zusammenhang auch häufig davon die Rede, dass es darum geht, für bestimmte, von der Politik erwünschte Maßnahmen, die notwendige „Akzeptanz" herzustellen. Die Beispiele dafür sind zahlreich und erstrecken sich auf alle Politikfelder. So ist in der Debatte um den Ausbau der Erneuerbaren Energien immer wieder zu hören, dass es darum gehe, bei den Bürgern die Akzeptanz für weitere Windparks in den hügeligen Landschaften der Mittelgebirge zu erhöhen oder dafür zu sorgen, dass sich der Widerstand gegen Stromtrassen in Verständnis für deren Notwendigkeit verwandelt. Genau dabei soll die Wissenschaft helfen. Eine Analyse der Frage, ob Windparks und Stromtrassen eigentlich eine rationale Form von Klimaund Energiepolitik sind, ist dagegen weit weniger erwünscht.

Vor diesem Hintergrund ist ziemlich klar, dass sich Politiker für Nudging interessieren. Die Verhaltensökonomen und die experimentelle Wirtschaftsforschung haben aus der Sicht der Politik so eine Art „Trickkiste“ ge- bzw. erfunden, in die zu greifen sehr verlockend ist. Endlich versprechen Ökonomen einmal genau das zu liefern, was die Politik am liebsten hätte. Gebrauchsanweisungen dafür, wie man die eigene Politik am besten dem Wähler (Bürger) verkauft und keine nörgeligen Analysen, die aufzeigen, dass die Politik mal wieder alles falsch macht. Das Standardbeispiel für "gelungenes Nudging" macht klar, wie es funktioniert: Die Politik wünscht sich mehr Organspender, weiß aber nicht, wie sie die Menschen dazu bewegen soll, endlich einen Organspenderausweis auszufüllen und mit sich zu führen. Die Verhaltensökonomen wissen, wie es geht. Anstatt die Menschen vor die Aufgabe zu stellen, sich aktiv als Organspender zu outen, stellt man sie vor die Aufgabe, nur für den Fall aktiv zu werden, dass sie keine Organe spenden wollen. Im Prinzip geht es immer um die gleiche Entscheidung: Organspender oder kein Organspender? Aber in erstem Fall hat man ein paar Prozent der Bevölkerung, die Organspender werden, im zweiten Fall sind es nur ein paar Prozent, die keine Organspender sein wollen. Ein einfacher Trick mit einem durchschlagenden Erfolg. Aber ist es auch ein guter Trick?

Die Politik verspricht sich vom Nudging mehr solcher Tricks. Schließlich stehen bockige Bürger nicht nur beim Organspenden auf der Leitung. Es gibt immer mehr Dinge, die die Politik will und die sich als NIMBY-Güter (Not In My Back Yard) entpuppen. Dabei geht es längst nicht nur um Atommüllendlager, Stromtrassen, Autobahnen und Ähnliches. Mittlerweile bilden sich auch Bürgerinitiativen gegen den Bau von Autobahn-Rastplätzen oder es kommt zu Bürgerbewegungen gegen den Neubau eines Bahnhofes. Da wäre es sehr hilfreich, ein paar Tricks auf Lager zu haben, die helfen, aus Wutbürgern einsichtige Zeitgenossen zu machen, die sich mit dem Unvermeidlichen abfinden. Soweit die Interessenlage der Politik. 


\section{Wissenschaft im Dienst der Gesellschaft}

Woran sollte die Wissenschaft interessiert sein? Das ist natürlich eine normative Frage, deren Beantwortung sich einer strikt wertfreien wissenschaftlichen Methodik entzieht. Deshalb sei an dieser Stelle darauf verwiesen, dass sich in der wissenschaftlichen Gemeinschaft in den letzten Dekaden ein allgemeiner Konsens in dieser Frage herausgebildet hat, auf den hier Bezug genommen wird. Dieser Konsens besteht darin, dass es unter anderem Aufgabe der Wirtschaftswissenschaft ist, die Erkenntnisse, zu denen sie gelangt, in den Dienst der Gesellschaft zu stellen. Durch Beratung der Politik gilt es dazu beizutragen, dass politische Entscheidungen besser fundiert werden können, rationaler werden. Sie sollten sich nicht an Ideologien, Irrglauben und Meinungen orientieren, sondern - soweit das möglich ist - an wissenschaftlich nachgewiesener Evidenz. Das ist zunächst einmal etwas vollkommen anderes als Nudging. Ist damit die Verhaltensökonomie als Methode zur Politikberatung ungeeignet? Wenn man sie mit Nudging gleichsetzt, dann könnte dieser Schluss naheliegen. Um zu zeigen, dass Verhaltensökonomie mehr ist als Nudging und dass sie gewinnbringend in der Politikberatung eingesetzt werden kann, muss man sich mit den Methoden der Politikberatung etwas näher befassen.

In der letzten Zeit ist unter deutschen Ökonomen sehr viel von „evidenzbasierter Politikberatung“ die Rede. Auf der letzten Jahrestagung des Vereins für Socialpolitik war sie Gegenstand der Kerntagung und die Hauszeitung des Vereins, die „Perspektiven der Wirtschaftspolitik“, veröffentlichte gleich zwei wichtige Beiträge zu diesem Thema innerhalb kurzer Zeit. ${ }^{1}$ Beim Studium dieser Beiträge entsteht der Eindruck, als sei klar definiert, was „evidenzbasiert“ bedeutet. Demnach ist darunter ausschließlich empirische Evidenz zu verstehen, die mit Hilfe ökonometrischer Methoden auf der Grundlage von Felddaten gewonnen wird. Es soll hier nicht daran gezweifelt werden, dass die so verstandene evidenzbasierte Politikberatung äußerst wichtig und verdienstvoll ist. Es gilt auch anzuerkennen, dass es in den letzten Dekaden enorme Fortschritte bei der Entwicklung ökonometrischer Methoden gegeben hat, die es heute erlauben, deutlich mehr wirtschaftspolitisch relevante Fragen fundiert und kompetent zu behandeln, als das noch vor 20 Jahren der Fall war. So klar das alles ist, so unklar ist dabei, welche Rolle die Bereiche unserer Disziplin spielen, die nicht in diesem Sinne Evidenz liefern. Konkret: Welche Rolle spielt die reine Theorie und welche Rolle spielen das Laborexperiment und die Verhaltensökonomik? Letztere werden in den genannten Beiträ-

1 Vgl. F. Kugler, G. Schwerdt, L. Wößmann: Ökonometrische Methoden zur Evaluierung kausaler Effekte der Wirtschaftspolitik, in: Perspektiven der Wirtschaftspolitik, 15. Jg. (2014), H. 2, S. 105-132; C M. Schmidt: Wirkungstreffer erzielen - Die Rolle der evidenzbasierten Politikberatung in einer aufgeklärten Gesellschaft, in: Perspektiven der Wirtschaftspolitik, 15. Jg. (2014), Nr. 3, S. 219-233. gen mit keinem Wort erwähnt. Bedeutet das, dass sie keine Rolle in der Politikberatung spielen sollten?

\section{Kausalanalyse als Königsdisziplin}

Die Laborökonomik und die Verhaltensökonomik auszuklammern, wäre ein schwerer Fehler. Um dies begründen zu können, bedarf es einer Evaluation der Methoden, einer Bewertung an einem einheitlichen Maßstab. Dieses Maß besteht aus zwei Kriterien, an denen sich Methoden hinsichtlich ihrer Eignung für die Politikberatung messen lassen. Das erste Kriterium betrifft die Fähigkeit, kausale Zusammenhänge nachweisen zu können. Völlig zu Recht schreibt Christoph M. Schmidt: „Ohne eine Einsicht in das Zusammenspiel von Ursache und Wirkung ist keine sinnvolle wirtschaftspolitische Weichenstellung möglich."2 Folgerichtig bezeichnet er die Kausalanalyse als die Königsdisziplin der Wirtschaftsforschung. Das zweite Kriterium bezieht sich auf die Fähigkeit einer Methode, Kausalitäten nicht nur zu identifizieren, sondern diese auch in der Realität mehr oder weniger sicher zu verorten. Angesprochen ist damit die sogenannte externe Validität wissenschaftlicher Aussagen. Es ist hier nicht der Raum, um ausführlich diese beiden Kriterien zu diskutieren, ${ }^{3}$ dennoch erlauben sie, auf recht einfache Weise eine Einordnung der wichtigsten Werkzeuge, derer sich die Ökonomik bedient.

Die reine Theorie benutzt axiomatische Systeme, innerhalb derer sich Kausalitäten zweifelsfrei nachweisen lassen - das ist ihr großer Vorteil. Innerhalb eines mathematischen Modells lassen sich aus den getroffenen Annahmen Schlussfolgerungen deduzieren, deren Richtigkeit mit der Genauigkeit eines mathematischen Beweises belegt werden kann. Eine transparentere Darlegung von kausalen Zusammenhängen ist nicht möglich. Die Königsdisziplin beherrscht die mathematische Wirtschaftsforschung perfekt. Nicht so gut sieht es im Hinblick auf die externe Validität aus. Ob die Zusammenhänge, die sich im Modell so glasklar zeigen lassen, in der Realität Bedeutung besitzen und dort wirksam sind, ist eine weitgehend offene Frage, mit deren Beantwortung sich Theoretiker lange Zeit kaum beschäftigt haben.

Genau umgekehrt verhält es sich bei der empirischen, auf Felddaten gestützten Forschung. Die Fähigkeit, Kausalität nachzuweisen, ist hier stark eingeschränkt. Sie ist es vor allem deshalb, weil für diesen Nachweis Identifikationsprobleme gelöst werden müssen, die sich oft genug als nicht lösbar erweisen. Zwar sind in dieser Hinsicht riesige methodische Fortschritte erzielt worden, dennoch bleiben die Möglichkeiten beschränkt. Wenn es darum geht, Kausalzusam-

2 Ebenda, S. 223

3 Vgl. dazu J. Weimann: Verhaltensökonomik und Politikberatung, erscheint in: M. Held, G. Kubon-Gilke, R. Sturn (Hrsg.): Reformen und ihre politisch-ökonomischen Fallstricke, Jahrbuch: Normative und institutionelle Grundfragen der Ökonomik, Bd. 14, 2015. 
menhänge, für die keine Felddaten zur Verfügung stehen, zu untersuchen, muss die empirische Forschung die Waffen strecken. Das ist eine triviale Einsicht, an der aber oft genug kein Weg vorbei führt. Beispielsweise ist dies regelmäßig der Fall, wenn es um die Evaluation von institutionellen Veränderungen geht, also um Institutionen, die noch nicht existieren und für die deshalb auch keine Beobachtungen vorliegen können. So eingeschränkt die Empirie bei der Kausalanalyse ist, so stark kann sie bei der externen Validität punkten. Leider aber auch nur dann, wenn sie die Identifikationsprobleme tatsächlich gelöst hat. Ist das nicht der Fall, können die Zusammenhänge, die empirisch nachgewiesen werden, tatsächlich als kausale Beziehung existieren - oder eben auch nicht. Christoph M. Schmidt weist sehr eindringlich darauf hin, dass es für die evidenzbasierte Politikberatung essenziell sei, ihre Erkenntnisgrenzen sehr klar offenzulegen.

\section{Verhaltensökonomie und Laborexperiment}

Bis hierher zeigt sich, dass es einen Trade-off zwischen der Fähigkeit, Kausalität nachzuweisen und der externen Validität, mit der eine Methode ausgestattet ist, gibt. Wie schneiden die Verhaltensökonomik und das Laborexperiment dabei ab? Für die empirischen Politikberater ist das kontrollierte Zufallsexperiment der „Goldstandard“" . Gemeint ist dabei aber das Feldexperiment, das die Politik oder eine glückliche Fügung mitunter den Forschern zur Verfügung stellt. Wenn solche Fügungen sich nicht ergeben, oder wenn es um hypothetische Institutionen geht, kann das Laborexperiment einspringen. Mit inm lassen sich Fragen beantworten, die der rein theoretischen und der empirischen Arbeit verschlossen sind. Im Labor lassen sich Institutionen schaffen und beliebige Entscheidungssituationen unter kontrollierten Bedingungen herstellen. Experimentelle Untersuchungen beziehen sich auf Treatment-Effekte, d.h. auf die Effekte, die durch systematische Variation einzelner Parameter des experimentellen Designs ausgelöst werden. Und das erlaubt eine Kausalanalyse, die in ihrer Qualität durchaus an die reine Theorie heranreicht. Das ist unbestritten. Vorbehalte gibt es hinsichtlich der externen Validität: Lernen wir aus Experimenten etwas darüber, was in der Realität passiert? Vor allem Theoretiker, aber auch Empiriker sind da skeptisch. Aber sie übersehen zwei Dinge: Erstens hat das Experiment bei annähernd gleichen Fähigkeiten bezüglich der Kausalanalyse gegenüber der Theorie notwendigerweise schon deshalb eine höhere externe Validität als das theoretische Modell, weil im Labor reale Menschen entscheiden und nicht artifiziell erzeugte Homines oeconomici. Zweitens zwingt uns der Trade-off zwischen Kausalanalyse und externer Validität, alle Möglichkeiten auszuschöpfen, die sich im Spannungsfeld dieser beiden Dimensionen ergeben. Auf die der Laborexperimente zu verzichten, ist kaum zu begründen.

4 Vgl. C. M. Schmidt, a.a.O., S. 226.
Die Substitution des Homo oeconomicus durch reale Menschen, die im Labor stattfindet, hat wesentlich zur Entstehung der Verhaltensökonomik beigetragen, die die Schablonen liefert, die beim Nudging benutzt werden. Aber die Verhaltensökonomik leistet viel mehr und sie leistet vor allem Dinge, die sich sehr gewinnbringend in die Art der Politikberatung einbringen ließen, wie sie zu Recht von führenden Ökonomen eingefordert wird. Zwei Beispiele seien genannt.

\section{Zwei Beispiele}

In der wirtschaftspolitischen Diskussion - und nicht nur dort - haben Auktionen in den letzten Dekaden sehr an Bedeutung gewonnen. Die Versteigerung von Emissionsrechten und die geplante Versteigerung von Kraftwerkskapazitäten sind zwei aktuelle Beispiele aus der Energiepolitik. Eine wirtschaftspolitische Beratung ist an dieser Stelle sehr sinnvoll und notwendig, denn das optimale Design von Auktionen zu finden, ist alles andere als eine triviale Aufgabe. Die umfangreiche experimentelle Forschung zum Auktionsdesign hat gezeigt, ${ }^{5}$ dass Modelle, in denen Bieter strikt rational handeln, allein keine guten Ratgeber sind, weil reale Bieter Dinge tun, die in den Modellen nicht vorkommen. Die empirische Forschung ist an dieser Stelle ohnehin machtlos, weil sich wesentliche Parameter einer Auktion (beispielsweise maximale Zahlungsbereitschaften) nicht beobachten lassen. Eine evidenzbasierte Politikberatung erzwingt in diesem Fall geradezu den Einsatz experimenteller Methoden.

Das zweite Beispiel ist gänzlich anderer Natur. Bei der Besteuerung gehen sowohl Politiker als auch Ökonomen in aller Regel davon aus, dass die Zensiten die Steuersätze unverzerrt wahrnehmen. Die experimentelle Forschung hat gezeigt, dass dies nicht so sein muss, sondern dass Menschen beispielsweise einer Art Nettolohnillusion unterliegen können. Im Ergebnis hat das zur Folge, dass die Arbeitsanstrengung unter Besteuerung zu hoch ausfällt, weil Beschäftigte Teile der Steuerzahlung irrtümlich als Teil ihres Nettoeinkommens ansehen. ${ }^{6}$ Wie geht man mit einer solchen Erkenntnis um? Eine Möglichkeit besteht darin, dass man mit Verweis auf diese Resultate die Steuern auf Arbeitseinkommen erhöht, weil die Zusatzlasten der Besteuerung tatsächlich geringer ausfallen, als theoretisch zu erwarten wäre. Das ist gewissermaßen die Nudging-Variante, bei der die Informationsasymmetrie zwischen Besteuerten und Politik ausgenutzt wird, um das Steueraufkommen zu erhöhen. Eine andere Möglichkeit wäre, nach Wegen zu suchen, diese Asymmetrie zu beseitigen, um den Menschen den Fehler zu

5 Vgl. dazu H.-T. Normann, R. Ricciuti: Laboratory Experiments for Economic Policy Making, in: Journal of Economic Surveys, 23. Jg. (2009), H. 3, S. 407-432

6 Vgl. M. Fochmann, J. Weimann: The Effects of Tax Salience and Tax Experience on Individual Work Efforts in a Framed Field Experiment, in: Finanzarchiv/Public Finance Analysis, 69. Jg. (2013), Nr. 4, S. 1-32. 
ersparen, für Geld zu arbeiten, das sie gar nicht bekommen. Das wäre die Variante, bei der wirtschaftspolitische Beratung im Sinne der Aufklärung eingesetzt wird. Entscheidend ist, dass Beratung dieser Art nur auf der Basis von Erkenntnissen der experimentellen Forschung möglich ist. Sie zu missachten, oder sie nur zum Zwecke des Nudgings zu (miss-)brauchen, ließe sich mit der Forderung nach einer strikt evidenzbasierten Politikberatung kaum vereinbaren.
Das Interesse der Bürger - um deren Wohlfahrt es letztlich geht - dürfte damit auch klar sein. Es wird sich darauf richten, wissenschaftliche Methoden zu nutzen, um einerseits der Politik dabei zu helfen, möglichst rationale Entscheidungen zu treffen und andererseits sie selbst vor Entscheidungen zu bewahren, die sie nur treffen, weil sie einer Informationsasymmetrie ausgesetzt sind. Das ist etwas anderes als Nudging.

\section{Marlene Haupt}

\section{Nudging im Bereich der Alterssicherung - warum und wie?}

In allen Industrienationen stellt die Alterssicherung den Kern der sozialen Sicherungssysteme dar, wenngleich bei der konkreten Ausgestaltung verschiedene Wege beschritten werden. Als Reaktionen auf sich ändernde soziale, politische, ökonomische und insbesondere demografische Rahmenbedingungen haben allerdings in den letzten zehn bis 15 Jahren viele Länder in diesem Kernbereich der sozialen Sicherung umfangreiche Reformen vollzogen. Häufig waren diese Reformen echte Paradigmenwechsel in der Alterssicherungspolitik, die zu einem strukturellen Umbau der Alterssicherungssysteme führten und häufig dem Drei-Säulen-Modell aus gesetzlicher, betrieblicher und privater Altersvorsorge folgten.

So erfolgte in Deutschland beispielsweise mit der Rentenreform von 2001 eine Abkehr vom Ziel der Lebensstandardund Statussicherung durch das System der gesetzlichen Rentenversicherung hin zu einer Politik des stabilen Beitragssatzes auf Basis eines Mehrsäulensicherungssystems aus gesetzlicher, betrieblicher und privater Altersvorsorge. Erreicht werden sollte dies zum einen durch die Absenkung des Rentenniveaus in der gesetzlichen Rentenversicherung aufgrund einer modifizierten Rentenanpassung und zum anderen durch die substanzielle Stärkung der ergänzenden betrieblichen und privaten Altersvorsorge. Da eine obligatorische kapitalgedeckte Zusatzvorsorge in der Öffentlichkeit allerdings auf massive Kritik stieß, sollte eine möglichst weite Verbreitung der freiwilligen Zusatzvorsorge durch staatliche Förderung, insbesondere in Form der sogenannten Riesterrente, erreicht werden.

Diese Reform und die abgeleitete Etablierung eines sogenannten Wohlfahrtsmarktes verlangen nun vereint als neues Leitbild der Alterssicherung von den Versicherten allgemein ein bis dato nicht notwendig gewesenes Maß an Eigenverantwortung für die Altersvorsorge bei gleichzeitiger Ermöglichung von mehr individueller Selbstbestimmung und Gestaltungsoptionen: In Deutschland kann und muss nun seit der Rentenreform von 2001 selbständig entschieden werden, ob und wie tatsächlich ergänzend zur gesetzlichen Rentenversicherung vorgesorgt wird und welche Produkte gewählt werden. Dieser marktbasierten Wahlfreiheit und damit verbundenen Entscheidungssouveränität des Individuums steht eine große Zahl von Anbietern, Anlageformen, Fördermöglichkeiten und Produkten der betrieblichen und privaten Altersvorsorge gegenüber, über die sich jeder eigenständig informieren muss.

\section{Verhaltensmodell des Homo oeconomicus}

Dieses theoretische Konstrukt der Konsumentensouveränität fußt wiederum auf dem der modernen neoklassischen Ökonomie zugrunde liegenden Verhaltensmodell des Homo oeconomicus ohne letztlich zu prüfen, inwieweit das tatsächliche Verhalten der Individuen auf Wohlfahrtsmärkten mit den ökonomischen Vorstellungen vom rational und eigennützig handelnden und vollständig informierten Konsumenten übereinstimmt. An dieser Stelle setzt schließlich die verhaltensökonomische Forschung an, deren zentraler Gegenstand nicht die pauschale Ablehnung neoklassischer Ansätze ist, sondern vielmehr die Überzeugung, dass eine Berücksichtigung psychologischer Grundlagen und die Modifizierung einzelner Annahmen des ökonomischen Standardmodells die Ökonomie zu ihren eigenen Bedingungen verbessern kann. ${ }^{1}$ Durch die Untermauerung der Ökonomie mit psychologischen bzw. sozialwissenschaftlichen Erkenntnissen sollen ihre eigenen theoretischen Grundlagen, Prognosefähigkeiten und Elemente der Politikberatung verbessert und der Realität, und damit auch dem tatsächlichen individuellen Verhalten, dem des Homo sapiens, angenähert werden. ${ }^{2}$

1 Vgl. C. F. Camerer, G. Loewenstein: Behavioral Economics: Past, Present, Future, in: C. F. Camerer, G. Loewenstein, M. Rabin (Hrsg.): Advances in Behavioral Economics, Russell Sage Foundation, New York u.a.O. 2004, S. 3-51; T. H. Ho, N. Lim, C. F. Camerer: Modeling the Psychology of Consumer and Firm Behavior with Behavioral Economics, in: Journal of Marketing Research, 43. Jg. (2006), H. 3, S. 307-331.

2 R. H. Thaler: From Homo Economicus to Homo Sapiens, in: The Journal of Economic Perspectives, 14. Jg. (2000), H. 1, S. 133-141. 
Zentraler Analysegegenstand für die Verhaltensökonomie sind Anomalien bzw. systematische Verzerrungen als Abweichungen und Unregelmäßigkeiten des tatsächlichen individuellen Verhaltens im Sinne von „psychologischen Normalfällen" gegenüber der Modellvereinfachung des Homo oeconomicus. In ihrer Methodik ist die Verhaltensökonomie dabei bereits seit ihren Anfängen sehr stark durch die psychologische Forschung, also durch empirische, häufig experimentelle Designs, geprägt, da sich diese Methoden als besonders wertvoll erwiesen haben, um verhaltens- und standardökonomische Erklärungsmuster voneinander abzugrenzen. ${ }^{3}$

\section{Anomalien bei der Altersvorsorge}

Nun sind aber wiederum nicht alle verhaltensökonomischen Erkenntnisse von Relevanz für den freiwilligen Spar- und Altersvorsorgeprozess. Im Folgenden wird daher eine Auswahl hierbei relevanter Anomalien bzw. systematischer Verzerrungen aufgezeigt, die keinesfalls den Anspruch erhebt, vollständig zu sein: ${ }^{4}$

- Verlustaversion: Die Bewertung von Gewinnen und Verlusten erfolgt dabei nicht absolut, sondern relativ zu einem Referenzpunkt, wobei Verlusten zudem ein deutlich höherer Wert beigemessen wird als Gewinnen in gleicher Höhe. Aus Angst vor finanziellen Verlusten kann eine freiwillige Altersvorsorge daher unterbleiben.

- Verzerrung des Status quo: Aus der Verlustaversion leitet sich eine Folgeanomalie ab, nach der Individuen eine starke Tendenz aufweisen im Status quo zu verharren, da die Nachteile, diesen zu verlassen, gravierender erscheinen als die Vorteile. Einmal getroffene Entscheidungen, wie die Wahl des abgeschlossenen Vertrages, werden entweder nicht mehr hinterfragt oder es kommt erst gar nicht zu einem Vertragsabschluss, da die Situation ohne private Zusatzvorsorge unbedenklicher erscheint.

- Informationspräsentation: Die Art der Informationspräsentation, d.h., ob und wie eine positive oder negative Darstellung einer Aussage erfolgt, beeinflusst die danach

3 Vgl. R. Sudgen: Experiment, Theory, World: A Symposium on the Role of Experiments in Economics, in: Journal of Economic Methodology, 12. Jg. (2005), H. 2, S. 177-184; B. S. Frey, A. Stutzer: Economics and Psychology: Developments and Issues, in: B. S. Frey, A. Stutzer (Hrsg.): Economics and Psychology: A Promising New Cross-disciplinary Field, Cambridge u.a.O. 2007; L. A. Reisch, A. Oehler: Behavioral Economics: Eine neue Grundlage für die Verbraucherpolitik, in: Vierteljahrshefte zur Wirtschaftsforschung, 78. Jg. (2009), H. 3, S. 30-43.

4 Die Forschung kennt mittlerweile über 100 verschiedene Anomalien. Hier kann nur eine Auswahl vorgestellt werden, die insbesondere im Bereich der (freiwilligen) Altersvorsorge relevant ist. Weitere Literatur zu Verhaltensabweichungen findet sich z.B. ab 1987 in den Sonderbeiträgen „Anomalies“ des Journal of Economic Perspectives sowie bei D. H. Enste, M. Hüther: Verhaltensökonomik und Ordnungspolitik. Zur Psychologie der Freiheit, IW-Positionen, Nr. 50, Institut der deutschen Wirtschaft, Köln 2011. getroffenen Entscheidungen. Bei der Fokussierung auf einen Gewinn neigen Individuen zu einer Risikoaversion, bei der Fokussierung auf einen Verlust zur Risikobereitschaft. Relevant ist auch die Reihenfolge der Informationspräsentation, also wann eine Information erhalten oder gegeben wird.

- Überflutung mit Wahlmöglichkeiten: Eine große Zahl an (Produkt-)Wahlmöglichkeiten erhöht die daraus resultierenden Kosten der Informationsbeschaffung deutlich. Bei den Individuen stellt sich schnell das Gefühl der Überforderung ein. Auch sinkt sowohl die Motivation, ein Produkt auszuwählen, als auch die Zufriedenheit mit der Entscheidung mit der Zahl der zur Auswahl stehenden Produkte. Bei der Altersvorsorge wird dann entweder gar keine Entscheidung getroffen und kein Vorsorgevertrag abgeschlossen oder die Informationssuche vorzeitig abgebrochen, keine Vergleiche durchgeführt und so möglicherweise ein nicht geeignetes Produkt gewählt.

- Finanz- und Altersaversion: Je umfangreicher und aufwendiger die Beschaffung, Verarbeitung und Auswertung von Informationen zur Bewältigung finanzieller Entscheidungen sind, desto eher kommt es zu einer Überforderung. Dies ist besonders im Zusammenhang mit geringer finanzieller Allgemeinbildung und wenig Erfahrung im Umgang mit Finanzprodukten ausgeprägt. Weiterhin wird der Zustand des Alters systematisch mit negativen Eindrücken und Altersklischees wie geringerer Produktivität, Einsamkeit, Armut, Krankheit und Pflegebedürftigkeit assoziiert. Ein Unterlassen der Beschäftigung mit dem Thema der privaten Altersvorsorge wird bei einer Abneigung gegenüber Finanzthemen und negativen Altersbildern somit wahrscheinlicher.

- Kurzsichtiges Verhalten und Gegenwartspräferenz: Individuen tendieren dazu, die in der entfernten Zukunft liegenden Konsequenzen ihres Verhaltens in der gegenwärtigen Situation nicht ausreichend zu berücksichtigen. Weiterhin werden gegenwärtige Bedürfnisse gegenüber zukünftigen bevorzugt. Da bei kaum einem Gut der Anlagehorizont so lang ist wie bei der Altersvorsorge, dominieren hier Gegenwartspräfenzen besonders - der gegenwärtige Konsum wird dem im Rentenalter vorgezogen.

- Mangelnde Selbstkontrolle: Diese Verletzung der intertemporalen Nutzenmaximierung entsteht durch ein Gefüge von lang- und kurzfristigen Präferenzen, die dazu führen, dass das Individuum als aus einem „Planer“ und einem „Macher" bestehend aufgefasst wird. Der „Macher“ hat einen kurzfristigen Zeithorizont und ist auf den gegenwärtigen Konsum fokussiert, während der „Planer“ auf die Nutzenmaximierung über den Lebenszyklus ausgerichtet ist. Alltäglichen Versuchungen und Verlockungen, 
denen der „Macher“ eher unterliegt, kann der „Planer“ durch persönliche Willensstärke begegnen. Auch kann er sich zusätzlich an Regeln binden, die die Konsummöglichkeiten des „Machers“ einschränken, z.B. durch den Abschluss eines festen Sparplans zur Altersvorsorge.

Da nun gerade die freiwillige Altersvorsorge zunehmend als komplexer und vielschichtiger Prozess betrachtet wird, der in eine Vorsorgeplanung und das eigentliche Vorsorgesparen unterteilt werden kann, ist hier die Konsumentensouveränität durch die dargestellten Abweichungen vom rationalen Verhalten im Sinne des Homo oeconomicus oft nicht in vollem Umfang gewährleistet. Denn erst wenn alle Prozessstufen der freiwilligen Altersvorsorge mit positivem Ergebnis durchlaufen werden, kommt es überhaupt zum Vorsorgesparen. Dabei ist es nicht unerheblich, dass je nach Entscheidungssituation andere Faktoren relevanter sind. Das bedeutet nichts anderes, als dass die Auseinandersetzung mit dem Thema Altersvorsorge und die Altersvorsorgebereitschaft z.B. durch andere Verhaltensanomalien bzw. systematische Verzerrungen beeinflusst wird, als die konkrete Auswahl des Produktes.

\section{Nudges in der Altersvorsorge}

Zur Ermöglichung der gewünschten Konsumentensouveränität im Bereich der freiwilligen Altersvorsorge können nun wiederum die von Thaler und Sunstein ${ }^{5}$ geprägten systematischen „Nudges“ eine Möglichkeit darstellen, diese Privatautonomie zu gewährleisten. Diese auch als libertärer Paternalismus bezeichneten „Nudges“ meinen Verhaltensanstöße, die ausdrücklich keine Anordnungen oder Verbote sind und ohne größeren Aufwand zu umgehen sein müssen. Angewendet auf den Bereich der Alterssicherung kann innerhalb der systematischen „Nudges“ als stärkstes Eingreifen ein Opt-out-Modell angesehen werden. Dabei erfolgt eine automatische Altersvorsorge, wenn nicht explizit widersprochen wird. Bedingt durch verschiedene Verhaltensanomalien (z.B. Trägheit und Status-quo-Verzerrung) beteiligen sich so tatsächlich mehr Individuen an Vorsorgesystemen als bei einem Opt-in-Modell wie der Riesterrente, bei dem aktiv ein Vertrag abgeschlossen werden muss. ${ }^{6}$

Im Sinne milderer bzw. weicherer Nudges kann im Bereich der privaten Altersvorsorge auch ein (staatliches) Basisprodukt als freiwillige Alternative zu privaten Anbietern etabliert werden. Spricht man den betroffenen Akteuren allgemein eine geringe Konsumentensouveränität zu, würde dies eine Möglichkeit darstellen, wie diese der Verhaltensanomalie der

5 R. H. Thaler, C. R. Sunstein: Nudge. Improving Decisions about Health, Wealth, and Happiness, New Haven, London 2008.

6 Vgl. J. J. Choi, D. Laibson, B. C. Madrian, A. Metrick: Saving For Retirement on the Path of Least Resistance, in: E. J. McCaffrey, J. Slemrod (Hrsg.): Behavioral Public Finance: Toward a New Agenda, Russell Sage Foundation, New York 2006, S. 304-351.
„Überflutung mit Wahlmöglichkeiten“ begegnen und auf einfache Art und Weise bei geringen Kosten der Informationsbeschaffung und -verarbeitung freiwillig für das Alter vorsorgen könnten. Allen anderen, d.h. den souveräneren Konsumenten, würde die Vielzahl der privaten Angebote aber weiter in vollem Umfang zur Auswahl stehen. Souveränere Individuen könnten auch selbst Vermeidungs- und Reduzierungsstrategien entwickeln, wenn sie sich der Existenz von Verhaltensanomalien bzw. systematischen Verzerrungen bewusst sind und diesen aktiv begegnen wollen. Beispielsweise könnten sich die Individuen durch den Abschluss eines Sparplans auf freiwilliger Basis in der Gegenwart verbindlich dazu verpflichten, in der Zukunft die Vorsorgeersparnis zu erhöhen. ${ }^{7}$

Eine Aufklärung über die insbesondere im Kontext des freiwilligen Altersvorsorgeprozesses auftretenden Verletzungen des Rationalitätsprinzips bzw. der intertemporalen Nutzenmaximierung könnte zusätzlich für diese Strategien sensibilisieren. Auch könnte eine begrenzte Auswahl möglicher Verträge angeboten werden, um die Informationsflut und eine Aversion gegenüber Finanzentscheidungen zu reduzieren, wobei der Abschluss alternativer Verträge natürlich weiterhin möglich sein müsste. Um der Anomalie der Altersaversion zu begegnen, könnte es hilfreich sein zu thematisieren, inwiefern ein ausreichendes Einkommen grundlegende Voraussetzung für eine aktive Teilhabe am gesellschaftlichen Leben im Alter ist und dass eine Beschäftigung mit diesen Themen damit unerlässlich ist, wenn es gleichsam möglich sein soll, lange unabhängig und selbständig zu leben, soziale Kontakte aufrechtzuerhalten und die Ruhestandsphase aktiv zu gestalten. $\mathrm{Zu}$ berücksichtigen ist jedoch, dass alle Möglichkeiten der Ausgestaltung privater Altersvorsorge unter Zuhilfenahme von „Nudges“ spezifische Vor- und Nachteile aufweisen, die von den politischen Akteuren bei einer konkreten Umsetzung und im Sinne des Grundsatzes der Verhältnismäßigkeit entsprechend abgewogen werden sollten. So nutzt eine Optout-Regelung zwar verschiedene Verhaltensanomalien, wie die Trägheit von Versicherten, aus und bindet so auch Individuen ein, die andernfalls keine aktive Vorsorge getroffen hätten. Die Forschung zeigt allerdings auch, dass viele Versicherte dann in diesem Standard verharren und sich keine Gedanken mehr über Alternativen machen. Möglicherweise attraktivere Anlagen werden so gar nicht mehr beachtet.

7 Neben der Idee des Opt-out, also dem automatischen Beitritt zu einem Altersvorsorgesystem, nennen R. H. Thaler, C. R. Sunstein, a.a.O., als eines der klassischen Beispiele für das „Nudging“ im Bereich der Alterssicherung die "Save-More-Tomorrow-Programme“, die R. H. Thaler, S. Benartzi: Save More Tomorrow: Using Behavioral Economics to Increase Employee Saving, in: Journal of Political Economy, 112. Jg. (2004), H. 1, S. S614-S187, entwickelt und in der Unternehmenspraxis empirisch überprüft haben. Ziel dieser Programme ist es, im Voraus automatische Beitragssteigerungen festzulegen (z.B. bei Lohnerhöhungen) und so die Sparquote sukzessive zu erhöhen. Natürlich ist auch immer eine Kombination von Opt-out-Regelungen und „Save More Tomorrow“ möglich. 
In Bezug auf die Etablierung eines Basisproduktes als zusätzliches Marktangebot oder auch auf die Einschränkung von Auswahlmöglichkeiten ist kritisch zu hinterfragen, welche Akteure letztlich darüber entscheiden dürfen, was angeboten und in welcher Form dies präsentiert wird. Die weicheren Verhaltensanstöße wie eine verbesserte Verbraucherinformationspolitik und die Stärkung des finanziellen Wissens sind bezüglich ihrer Effektivität letztlich schwer zu bewerten. Darüber hinaus zeigt sich in der praktischen Umsetzung häufig ein Problem der Selbstauswahl - bestimmte Verbrauchergruppen sind mit diesen Maßnahmen weiterhin schwer erreichbar. Auch kann das Aufschieben von Altersvorsorgeentscheidungen durch verbesserte Informationen und Verbraucherbildung nicht vollständig verhindert werden.

Interessant ist nun insbesondere im Bereich der Alterssicherung die Öffnung der Politik gegenüber verhaltensökonomischen Denkansätzen. So findet sich beispielsweise in einem Bericht der Bundesregierung zur Flexibilisierung des Über- gangs vom Erwerbsleben in den Ruhestand der Hinweis, dass es mit den Wertguthaben zwar seit längerem ein juristisches Instrument gibt, das dies ermöglichen könnte, dessen tatsächliche Verbreitung aber noch weit hinter den Erwartungen zurückbleibt. Dies wird sowohl mit der unzulänglichen Bekanntheit als auch der nicht ausreichenden Attraktivität für die Arbeitgeber begründet. Unter Berufung auf die Erkenntnisse der Verhaltensökonomie bedeutet dies konkret, dass es also nicht ausreicht, eine gesetzliche Regelung zu verabschieden. Es muss auch immer dafür gesorgt werden, dass diese in der Praxis angewendet wird. Darüber hinaus wird im Falle von Entscheidungsspielräumen der Arbeitgeber empfohlen, die gesellschaftlich gewünschte Alternative als Normalfall zu definieren, die immer dann greift, wenn nicht ausdrücklich eine andere Entscheidung getroffen wird. So müsste die wünschenswerte Variante, z.B. die Übertragbarkeit der Wertguthaben auf einen neuen Arbeitgeber, immer besonders attraktiv ausgestaltet werden und aus Unternehmenssicht am leichtesten umsetzbar sein, um letztlich Verhaltensänderungen anzustoßen.

\section{Lothar Funk}

\section{Sanfter Paternalismus und verhaltensökonomisch fundierte Ordnungspolitik im Verbraucherschutz}

Bei vielen liberalen Ökonomen stößt die wirtschaftspolitische Umsetzung verhaltensökonomischer Erkenntnisse nach wie vor auf erhebliche Vorbehalte. Allerdings lassen sich bei einer zunehmenden Zahl auch liberaler Ökonomen Tendenzen erkennen, die hierdurch ermöglichten Erkenntnisgewinne in wirtschaftspolitische Schlussfolgerungen umzumünzen. Spätestens seit der Finanzmarktkrise und der im Anschluss daran erfolgenden Kritik an der Beratungspraxis der Banken steht die Verbraucher- bzw. Verbraucherschutzpolitik in vielen Ländern und nicht zuletzt auch in Deutschland auf dem Prüfstand. Das ursprüngliche wirtschaftsliberale Leitbild des „mündigen Verbrauchers" wird nicht selten in Frage gestellt und durch einen neuen Ansatz des ,verletzlichen Verbrauchers" ergänzt oder ersetzt. ${ }^{1}$ Eine mitunter angenommene „strukturelle Asymmetrie“2 zwischen Konsumenten und Produzenten ist hier ein möglicher Ausgangspunkt für den vermehrten Einsatz des staatlichen verbraucherpolitischen Instrumentariums zur Korrektur dieses vermuteten Problems. Die aktuell hierzu vertretenen Positionen bleiben zumindest in Teilen unversöhnlich. ${ }^{3}$ Gerade im Zusammenhang mit der

1 Vgl. C. Bala, K. Müller (Hrsg.): Der verletzliche Verbraucher. Die sozialpolitische Dimension der Verbraucherpolitik, Düsseldorf 2014.

2 Vgl. R. Maier-Rigaud: Editorial: Verbraucherpolitische Entwicklungen und Herausforderungen in der Sozialpolitik, in: Sozialer Fortschritt, 62. Jg. (2013), H. 2, S. 41.

3 Vgl. hierzu den Überblick bei L. Funk: Die Themen im Sommer 2013, in: Das Wirtschaftsstudium, 42. Jg. (2013), H. 7, S. 907 f.
Frage nach der Integration verhaltensökonomischer Ansätze in die Wirtschaftstheorie wird aber nicht selten sowohl die Chance als auch die Notwendigkeit für eine verbesserte Verbraucher(schutz)politik gesehen, wenngleich die Einzelheiten der Ausgestaltung zwischen verschiedenen Denkschulen sehr kontrovers bleiben. ${ }^{4}$

Es stellen sich unter anderem die folgenden Fragen: Welche zentralen Verhaltensannahmen unterstellt der wirtschaftsliberale Mainstream und welche verbraucherpolitischen Schlussfolgerungen zieht er? Warum ist die Skepsis bei groBen Teilen der Wirtschaftsliberalen noch immer hoch, neuere Erkenntnisse der Verhaltensökonomik bei ihren politischen Schlussfolgerungen zu integrieren, und sind die Einwände von dieser Seite wirklich alle stichhaltig? Welche wesentlichen Implikationen ergeben sich aus einer verhaltensökonomisch fundierten Ordnungspolitik für die Verbraucherpolitik als einem zentralen Feld ihrer Anwendung?

\section{Neoklassisch-wohlfahrtsökonomischer Ausgangspunkt}

Basis der traditionellen neoklassisch-wohlfahrtsökonomisch ausgerichteten Theorie der Wirtschaftspolitik ist der

4 F. Rischkowsky: Verbraucherschutz im Binnenmarkt: Mehr als nur Wettbewerbspolitik?, in: Wirtschaftsdienst, 90. Jg. (2010), Sonderheft, S. 60; R. Maier-Rigaud, a.a.O., S. 42. 
Ansatz des Marktversagens und der Marktmängel, aus denen sich prinzipiell die gesellschaftliche Wohlfahrt steigernde korrigierende Staatseingriffe ableiten lassen. Anlässe hierfür können insbesondere Märkte mit unvollkommener Konkurrenz, das Auftreten von Externalitäten im Konsumbereich und das Problem unvollkommener Information der Verbraucher sein. ${ }^{5}$ Anhänger dieser Richtung können sehr klar vorhandene Zielkonflikte aufzeigen, die mit mehr oder weniger interventionistischen Eingriffen des Staates in der Wirtschaftspolitik generell und speziell beim Verbraucherschutz verbunden sind. Beispielsweise kann ein fürsorglicher Staat zwar seinen Bürgern ein Entscheidungsrisiko in Bezug auf Konsumauswahl und Informationskosten abnehmen, indem er hohe Produktstandards gesetzlich festlegt, nimmt aber dabei in Kauf, „dass durch die mit einer solchen Politik verbundenen höheren Preise einkommensschwache Verbrauchergruppen vom Konsum der betreffenden Güter ausgeschlossen sind“. 6

Aus dieser neoklassisch-wohlfahrtsökonomischen Sicht ist es mit der Fürsorge des Staates in Wirklichkeit nicht weit her, soweit hohe Produktstandards zu einer Rationierung über den Preis gerade von einkommensschwachen Verbrauchern führen. Folglich plädieren Anhänger dieser Richtung vorrangig für eine die Funktionsfähigkeit von Märkten sichernde Wettbewerbspolitik, staatliche Transferzahlungen für Bedürftige und für eine Verbraucherpolitik, die gesetzliche Auflagen und Gebote möglichst vermeidet und Bürgern mehr Eigenverantwortung auch in ihrem längerfristigen wohl verstandenen Eigeninteresse abverlangt. Dies erlaubt es den Bürgern, so die Begründung, ihrer Risikopräferenz und ihren persönlichen Lebensumständen entsprechend höhere Risiken bei geringeren Produktpreisen einzugehen, solange sie dies bewusst und bei voller Aufklärung tun, was eine höchstmögliche Effizienz der Konsumentscheidungen ermöglichen würde. ${ }^{7}$

\section{Wirtschaftsliberale Perspektiven}

Die informationsökonomische Erweiterung dieses Ansatzes bemängelt an diesem Grundmodell zwar sein Versagen in bestimmten Situationen, akzeptiert allerdings, dass erst dann ein staatlicher Eingriff zum Verbraucherschutz gerechtfertigt ist, ,wenn marktliche Mechanismen bei der Erhöhung der Funktionsfähigkeit von Märkten mit asymmetrischen Informationsstrukturen versagen"8. Tatsächlich können sich auch marktintern qualitätssichernde Instituti-

5 Vgl. zu Einzelheiten M. Ahlheim, A. Zahn: Versagt die Verbraucherpolitik?, in: Wirtschaftsdienst, 91. Jg. (2011), H. 3, S. 155-159.

6 Ebenda, S. 158.

7 Ebenda; und vgl. F. Rischkowsky, a.a.O., S. 60.

8 F. Rischkowsky, T. Döring: E-Commerce und europäische Verbraucherpolitik, in: Wirtschaftsdienst, 84. Jg. (2004), H. 5, S. 318. onen herausbilden, etwa durch Garantieversprechen von Firmen und Markenbildung. Folglich zielt diese Ergänzung des theoretischen Fundaments der Verbraucherschutzpolitik darauf $\mathrm{ab}$, besser funktionierende Informationsmärkte zu schaffen. Vorhandene Informationsasymmetrien sind abzubauen und der Informationsstand des Verbrauchers, der als schlechter informiert eingeschätzt wird, ist etwa über Informationspflichten der Anbieter oder ein Verbot irreführender Werbung sowie die Durchführung bzw. Subventionierung vergleichender Warentests zu verbessern, wenn der Markt zu wenig oder nur intransparente Informationen zur Verfügung stellt. ${ }^{9}$

Eine Hauptgemeinsamkeit beider heute als wirtschaftsliberal einzustufenden Perspektiven besteht darin, dass ursprünglich als Fundament für diese Analysen in aller Regel die Annahme rationalen Verhaltens - also „das Gedankenkonstrukt eines frei wählenden, tauschenden und rational handelnden Homo oeconomicus"10 - unterstellt wird. Dieser Ansatz findet sich in jedem volkswirtschaftlichen Einführungslehrbuch und hat sich angesichts seiner flexiblen Einsetzbarkeit auch vielfach als erster Erklärungsund Prognoseansatz bewährt. Er kann erfolgreich erweitert bzw. modifiziert werden, um seinen Anwendungsbereich noch auszuweiten. Vereinfacht wird im Basismodel individuelles Eigeninteresse sowie eine Orientierung der Menschen am subjektiv empfundenen Nutzen angenommen. Dabei wird jeweils eine Maximierung des individuellen Nutzens unter Berücksichtigung des ökonomischen Prinzips unterstellt. Allgemeiner ausgedrückt lässt sich das ökonomische Verhaltensmodell, das auf dem Leitbild des normativen Individualismus beruht, wie folgt kennzeichnen: „Die Trennung zwischen Präferenzen und Restriktionen, die Bewertung (eines Teils) der Alternativen, unter den bewerteten Alternativen die Entscheidung gemäß dem relativen Vorteil und damit die Beeinflussbarkeit dieses Verhaltens durch veränderte Umweltbedingungen (Anreize)." ${ }^{11}$

\section{Ablehnung paternalistischer staatlicher Politik}

Ein besonders hervorzuhebender gemeinsamer wirtschafts- und verbraucherpolitischer Nenner aus der Annahme rationalen Verhaltens besteht darin, (de)meritorische, bevormundende staatliche Eingriffe prinzipiell abzulehnen. Meritorische Bedürfnisse sind dadurch gekennzeichnet, dass das einzelne Individuum bzw. ein privater Haushalt aus Unwissenheit oder Entscheidungsunfähigkeit den Wert von bestimmten Gütern oder Dienstleistun-

9 Ebenda.

10 W. Sesselmeier, L. Funk, B. Waas: Arbeitsmarkttheorien. Eine ökonomisch-juristische Einführung, 3. Aufl., Heidelberg 2010, S. 74.

11 G. Kirchgässner: Homo Oeconomicus, 4. Aufl., Tübingen 2013, S. 32 f. 
gen falsch einschätzt und deshalb meritorische Güter (etwa Schulbildung) zu wenig nachfragt und folglich Anreize durch die Änderung der relativen Preise gesetzt werden müssten, um den Umfang der Nachfrage zu steigern (etwa durch Staatseingriffe verbilligter Zugang zu öffentlichen Bildungseinrichtungen), um eine als zu gering erachtete Nachfrage nach Bildung zu verhindern. Zudem fragt der Konsument nach diesem Konzept wegen der falschen individuellen Einschätzung seiner wahren Präferenzen zu viele sogenannte demeritorische Güter - wie etwa ungesunde Lebensmittel - nach, wovon er im eigenen langfristigen Interesse abgehalten werden sollte.

Offensichtlich widerspricht dies der für Marktwirtschaften zentralen Hypothese der Konsumentensouveränität und folglich auch der Idee des mündigen Verbrauchers..$^{12}$ Indem der Meritorik-Ansatz dem Staat eine bessere Kenntnis der tatsächlichen Individualinteressen zubilligt als den betroffenen Individuen selbst „und zu willkürlichen Eingriffen in die freie Konsumwahl missbraucht werden"13 kann, wurde er vielfach und insbesondere von Wirtschaftsliberalen als nicht ordnungskonform eingeschätzt. Doch hier existiert nicht nur eine Gegenbewegung, die das Meritorik-Konzept in den Mittelpunkt ihrer Analysen stellt. ${ }^{14}$ Zunehmend gibt es ebenfalls empirisch begründete Einwände gegen das im wirtschaftsliberalen Modell unterstellte menschliche Verhaltensmodell rationalen Handelns. Denn entscheidungstheoretische Untersuchungen haben eine Reihe von systematischen Abweichungen des tatsächlichen menschlichen Verhaltens vom Homo-oeconomicus-Ansatz aufgezeigt, etwa den Einfluss irrelevanter Informationen auf Entscheidungen, die häufige Fehleinschätzung von Wahrscheinlichkeiten oder die Minderschätzung künftiger Bedarfe. ${ }^{15}$ All dies ließ auch Teile der Wirtschaftsliberalen nicht unberührt und führte dort zu einem Forschungsprogramm einer „verhaltensökonomisch fundierten Ordnungspolitik"16.

12 Vgl. H. Luckenbach: Theoretische Grundlagen der Wirtschaftspolitik, 2. Aufl., München 2000, S. 173; vgl. auch kritisch hierzu B. Dinter, L. Funk, S. Pagel, in: C. Bala, K. Müller (Hrsg.), a.a.O., S. 128 f.

13 H. Luckenbach, a.a.O.

14 „Die Meritorik ist letztlich der Versuch, im Rahmen der ökonomischen Theorie dem Markt seine dienende, der Demokratie untergeordnete Rolle zuzuweisen. Demokratisch in Wahlen generierte gesellschaftliche Werturteile sollen demnach auch funktionsfähige Märkte einschränken und regulieren können. Insofern ist Meritorik nicht zwangsläufig paternalistisch, sondern eine Anerkennung des Primats demokratisch legitimierter Politik" (vgl. R. Maier-Rigaud, a.a.O., S. 41).

15 Hiervon wurde bis vor wenigen Jahren in der Volkswirtschaftslehre weitgehend abstrahiert, obgleich gewisse Modifizierungen des Rationalverhaltens bereits Mitte der 1950er Jahre erfolgten, insbesondere indem angenommen wurde, dass schon hinreichend akzeptable Ergebnisse durch die Akteure akzeptiert werden - Satisfizierung statt Optimierung (vgl. G. Kirchgässner, a.a.O., S. 32).

16 D. Enste, M. Hüther: Verhaltensökonomik und Ordnungspolitik. Zur Psychologie der Freiheit, Köln 2011, S. 82.

\section{Aktuelle wirtschafts- und verbraucherpolitische Strömungen im Vergleich}

Es kann sowohl nach Ansicht der Verfechter der Meritorik bzw. aus der Perspektive eines „liberalen Paternalismus“17 und aus einer verhaltensökonomisch fundierten wirtschaftsliberalen Sicht sinnvoll sein, wenn der Staat Menschen durch entsprechende Änderungen der Anreize in eine langfristig bessere Richtung lenkt. ${ }^{18}$ Dabei liegt ein paternalistischer Eingriff bereits vor, wenn durch staatliche Informationspolitik Informationsasymmetrien überwunden werden, wodurch Individuen zu ihrem eigenen Wohl beeinflusst werden sollen. ${ }^{19}$ Jüngst hat allerdings vor allem der liberale Paternalismus verstärkt Aufmerksamkeit auf sich gezogen. ${ }^{20}$ „Während ... der harte Paternalismus bestimmtes Handeln vollständig unterbinden will, erschwert es der weiche Paternalismus ,nur' [etwa über Besteuerung], während der sanfte Paternalismus AnstöBe zum richtigen Handeln über eine Beeinflussung der Informationsaufnahme bewirken will." ${ }^{21}$ Unter liberalem oder sanftem Paternalismus versteht man folglich „staatliche Regelungen, die auf direkten Zwang verzichten, aber Entwicklungen begünstigen, die zu höherer Lebenszufriedenheit führen“22. Ausgangspunkt hierfür ist die empirisch gut bestätigte Erkenntnis von Verhaltensökonomen, dass Menschen häufig unter anderem durch Willensschwäche gekennzeichnet sind, die zu Zeitinkonsistenzen des individuellen Verhaltens führen und den Status quo beibehalten wollen, auch wenn eine Situation willkürlich zustande gekommen ist und Veränderungen sowohl individuell wie auch für die Gesellschaft die Wohlfahrt steigern würden. Unser Verhalten hängt demnach nicht selten von solchen „Voreinstellungen“ und „Vorgaben“ oder „Defaults“ ab. ${ }^{23}$

„Nudging“ (Stupsen) setzt darauf, allein durch „eine kluge Architektur des Entscheidungsumfelds, z.B. über das Setzen der Voreinstellungen ... oder die Vereinfachung

17 Vgl. den Überblick bei L. Funk: Die Themen im Winter 2014, in: Das Wirtschaftsstudium, 43. Jg. (2014), H. 1, S. 67 f.; vgl. B. Kuhn: Verhaltensökonomische versus neoklassische Wirtschaftspolitik, in: Das Wirtschaftsstudium, 42. Jg. (2013), H. 5, S. $691 \mathrm{ff}$.

18 Vgl. ebenda, S. 692; L. Funk: Von Deutschland lernen - ja, aber anders, in: Finanz und Wirtschaft, Nr. 29 vom 14.4.2013, S. 2.

19 Vgl. G. Kirchgässner, a.a.O., S. 273. Informationsökonomisch begründete Verbraucherschutzpolitik beinhaltet demnach schon paternalistische Elemente.

20 Vgl. vor allem die grundlegende Einführung von R. H. Thaler, C. R. Sunstein: Nudge. Wie man kluge Entscheidungen anstößt, Berlin 2009.

21 G. Kirchgässner, a.a.O., S. 273.

22 J. Weimann, A. Knabe, R. Schöb: Geld macht doch glücklich, Stuttgart 2012, S. 81.

23 „Die Menschen sind oft schlichtweg zu bequem etwas zu ändern, oder unsicher, welche Folgen eine bestimmte Entscheidung haben könnte. Zum anderen sind viele Menschen von der Informationsflut überfordert und bleiben einfach bei ihren Gewohnheiten - oder überlassen anderen die Entscheidung." Zitiert nach Institut der deutschen Wirtschaft (Hrsg.): Keine Entscheidung ist auch eine, in: IW-Dienst, H. 48/2013 vom 28.11.2013, S. 6. 
des Zugangs zu alternativen Verhaltensweisen“ zu besseren Entscheidungen der Menschen beizutragen, ohne ihre Wahlmöglichkeiten einzuschränken, „da voreingestellte Alternativen auch abgelehnt werden könnten“24. Beispiele für „sanftes Schubsen“ wären etwa, in öffentlich betriebenen Mensen durch eine gezielte Anordnung der Speisen zu besseren Ernährungsgewohnheiten beizutragen oder durch Voreinstellung von Druckern, Papier normalerweise beidseitig und mit zwei Seiten pro Blatt zu bedrucken.

Der liberale Paternalismus beruht einerseits auf einer Verknüpfung von Neoklassik und Wirtschaftspsychologie. Er ist jedoch auch eine Reaktion auf erhebliche Angriffe, mit denen die traditionelle Neoklassik als wesentliche Basis wirtschaftsliberaler Argumentation weltweit und die vorrangig in Deutschland bedeutende liberale Ordnungspolitik konfrontiert sind. So ist die Neoklassik und deren wirtschaftspolitische Umsetzung als Neoliberalismus schon lange dem Ideologie- und Dogmatismusverdacht ausgesetzt, wonach sie häufig nicht den gesellschaftlichen Allgemeininteressen dienen, sondern vorrangig den Partikularinteressen der ökonomisch und politisch herrschenden Klasse nützen würden. ${ }^{25}$ Allerdings ist diese Kritik selbst ebenfalls ideologieverdächtig, da sich der neoklassische Denkansatz prinzipiell durchaus bewährt hat und auch heute noch sehr fruchtbar einsetzbar ist. Denn gerade die umstrittene Annahme strikt rationalen Verhaltens hilft dabei zu verstehen, „dass die Anreize der Dreh- und Angelpunkt der ökonomischen Analyse sind“ und sie bewahrt „die Akteure vor naiver Kooperation, ... die letztlich nur Enttäuschung nach sich zieht. Stattdessen wird der Blick darauf gelenkt, wie man bestimmte Regeln anreizkompatibel verankern kann" 26 .

Es erscheint jedoch ebenfalls abwegig, verhaltensökonomische Erkenntnisse und sich daraus ergebende strategische Interaktionen im Rahmen der Ordnungsökonomik einfach zu ignorieren, obwohl ihre aktive Berücksichtigung bei staatlichen Markt fördernden Eingriffen zu gesellschaftlichen Vorteilen ohne (nennenswerten) individuellen Freiheitsverlust führen könnte. Folglich verwundert es nicht, dass auch Teile der Wirtschaftsliberalen diese Befunde aufgreifen und ihre wirtschafts- und verbraucherpolitischen Implikationen entsprechend modifizieren, weil in der Vergangenheit allzu oft wirtschaftspsychologische Erkenntnisse vernachlässigt worden sind. Dies gilt etwa für das empirisch bestätigte Wissen, dass

24 Vgl. Stiftung Marktwirtschaft (Hrsg.): Mit Nudging zur Energiewende? (Tagungsbericht), Berlin 2013, S. 2.

25 Vgl. H. Trabold: Kapital. Macht, Politik. Die Zerstörung der Demokratie, Marburg 2014, S. $248 \mathrm{ff}$.

26 M. Leschke: Karl Homanns Ökonomik der Gesellschaftstheorie, in: I. Pies, T. Schönwälder-Kuntze, C. Lütge, A. Suchanek (Hrsg.): Freiheit durch Demokratie - Festschrift für Karl Homann, Berlin 2008, S. 209 vgl. dazu ebenfalls W. Sesselmeier, L. Funk, B. Waas, a.a.O., S. $89 \mathrm{ff}$.
Informationsüberflutung die individuelle Entscheidungsfindung oft nicht verbessert, sondern sogar nicht selten zu weniger rationalem Handeln führen kann. Im Ansatz verhaltensökonomisch fundierter Ordnungspolitik geht es daher darum, „durch staatliche Intervention die Nutzung der Freiheit für die Menschen einfacher und leichter zu machen, ohne ihre Freiheit materiell einzuschränken“27. Dennoch bleibt aber selbst der sanfte Paternalismus für viele eher traditionelle Wirtschaftsliberale offensichtlich äußerst problematisch, wie das folgende Zitat aus einem weit verbreiteten Lehrbuch zur Einschätzung von „wohlmeinender Bevormundung“ durch „sanfte“ Anstöße zu bestimmten erwünschten Handlungen verdeutlicht: „Offen bleibt ..., wer wodurch zu einer sanften Bevormundung der Bürger legitimiert ist. Auch eine wohlmeinende Bevormundung kann Freiheitsspielräume einengen, insbesondere dann, wenn die Maßnahmen mit Kosten verbunden sind, die auf die Allgemeinheit umgelegt werden. Schließlich wird gegen das Konzept eingewandt, dass paternalistische Verhaltenssteuerung unter Umständen eine Verminderung der Fähigkeit zu selbständiger Problemlösung bzw. zu souveräner Entscheidung nach sich zieht, was im Widerspruch zu Grundannahmen des ökonomischen Ansatzes steht. Als Schlussfolgerung ergibt sich, dass auch der Versuch der Beeinflussung menschlichen Verhaltens durch sanfte Anstöße das grundlegende Rechtfertigungsproblem nicht lösen kann.."28

\section{Viele Gegenargumente lassen sich entkräften}

Die Verfechter des verhaltensökonomisch fundierten Ordoliberalismus räumen zwar ein, dass der ursprüngliche „Nudging-Ansatz“ zwar mitunter bedenkliche, die Freiheit beschränkende interventionistische Elemente enthalte. Allerdings könnten diese Elemente aus dem Konzept herausgenommen werden. Per saldo ließe sich künftige Wirtschaftspolitik sehr wohl besser fundieren. ${ }^{29}$ Auch erscheinen die angeführten und weitere Kritikpunkte keineswegs als so stichhaltig, wie die Verfechter traditioneller Ordnungspolitik, die auf die Einbettung verhaltensökonomischer Erkenntnisse verzichten, nicht selten suggerieren. Zwar sind einige Kritikpunkte gegen den sanften Paternalismus durchaus gerechtfertigt, etwa dass dessen Verfechter bisweilen überzogene Erwartungen über den Erfolg solcher Maßnahmen wecken. ${ }^{30}$ Allerdings lassen sich viele auf Verhaltensökonomie beruhende Schlussfolgerungen sehr wohl auch wohlfahrtsökonomisch ableiten, etwa über hierarchisch geordnete Präferenzen, auch wenn dies oft bestritten wird. Die Be-

27 D. Enste, M. Hüther, a.a.O., S. 46.

28 M. Fritsch: Marktversagen und Wirtschaftspolitik, 9. Aufl., München 2014, S. $320 \mathrm{f}$

29 D. Enste, M. Hüther, a.a.O., S. 44 f.

30 Vgl. zum Folgenden G. Kirchgässner, a.a.O., S. 282 ff. 
hauptung, dass durch „Nudging“ die Fähigkeit zu selbstständiger Problemlösung gemindert werden könne, dürfte insofern zu pauschal sein, als ja auch „Lernen“ durch eine geänderte Entscheidungsarchitektur erst ermöglich werden kann. Die Neigung bei den Kritikern ist groß, zurückhaltende paternalistische Eingriffe mit idealtypischen Situationen zu vergleichen, statt mit dem Status quo, der oft durch staatliche Ge- und Verbote gekennzeichnet ist (also einem harten Paternalismus), was unangemessen erscheint. Problematisch erscheint neben anderen hier nicht weiter vorgestellten Punkten schließlich auch, dass einige wirtschaftsliberale Kritiker bei ihren Argumenten gegen die Erweiterungen der traditionellen Ordnungspolitik übersehen, dass sie sich dabei selbst das Recht anmaßen, „autoritativ darüber bestimmen zu wollen, welche Entscheidungen die Bürgerinnen und Bürger kollektiv über den politischen Prozess treffen dürfen und welche sie dem Markt überlassen sollten ${ }^{31}$.

Weitere Konflikte zwischen Verfechtern liberaler und verhaltensökonomisch inspirierter Ordnungspolitik sind angesichts dieser unterschiedlichen Einschätzungen vorprogrammiert. Die traditionelle, nicht verhaltensökonomisch fundierte Meritorik, durch die die von Konsumenten geäußerten Präferenzen durch andere Instanzen ergebnisbezogen korrigiert werden sollen, haben wirtschaftsliberale Anhänger der wohlfahrtsökonomisch begründeten Theorie des Marktversagens normalerweise abgelehnt und bleiben dabei bis heute. ${ }^{32}$ Dies gilt in der Regel ebenfalls für die Anhänger einer verhaltensökonomisch fundierten Ordnungspolitik. Allerdings haben sie im Vergleich sowohl gegenüber der ursprünglichen wirt-

31 G. Kirchgässner, a.a.O., S. 285.

32 „Der Verweis auf, übergeordnete“ Belange ist mit dem individualistischen Charakter des ökonomischen Ansatzes unvereinbar, denn im Rahmen der ökonomischen Sozialtheorie sind letztendlich die Präferenzen der Individuen maßgeblich bei der Beurteilung der Erwünschtheit von Zuständen bzw. für politische Ziele." Zitiert nach M. Fritsch, a.a.O., S. 319. schaftsliberalen Theorie der Wirtschaftspolitik wie auch gegenüber den Anhängern des liberalen Paternalismus einen erheblichen Vorteil in ihrer Positionierung. Indem sie die zusätzlichen Erkenntnisse aus der Verhaltensökonomik normativ umsetzen, sehen sie einerseits per saldo einen größeren Spielraum für wohlfahrtssteigernde Politik als typische liberale Ordnungspolitiker. Durch die strikte Minimierung freiheitsbeschränkender Eingriffe in dieser Konzeption, die im liberalen Paternalismus bisweilen nicht gewährleistet ist, beinhaltet dieser Ansatz andererseits ebenfalls wichtige Merkmale des traditionellen Wirtschaftsliberalismus, indem er gegenüber Gefahren eines möglichen Politikversagens viel wachsamer ist als der eindeutig staatsgläubigere „liberale“ Paternalismus. Darüber hinaus lässt sich dieses Forschungsprogramm insbesondere auch fruchtbar auf dem Gebiet einer problemorientierten und realitätsgerechteren Verbraucherpolitik anwenden, da die eindeutig zu pauschale Annahme eines vollständig rationalen Verhaltens der Wirtschaftsakteure aufgegeben und anerkannt wird, dass sich Informationsasymmetrien keineswegs immer mit mehr Informationen überwinden lassen. ${ }^{33}$ Die verbraucherpolitische Debatte wird fraglos durch verhaltensökonomische Erkenntnisse belebt, „als damit ein möglicher ,dritter Weg‘ zwischen den beiden Polen der reinen Freiheitssicherung einerseits und der bloßen Ergebnissteuerung andererseits aufgezeigt wurde." ${ }^{34}$ Erste Praxiserfolge durch staatliches „Nudging" etwa bei einer eindeutig verbraucherfreundlicheren Gestaltung der Voreinstellungen zu Zusatzleistungen bei Internetkäufen von Flügen (z.B. Verbot einer automatisch angeklickten Reiserücktrittsversicherung) sind zudem kaum zu leugnen.

$33 \mathrm{Vgl}$. zu einer ersten umfassenderen auch ordnungsökonomisch fundierten Analyse hierzu T. Döring, F. Rischkowsky: Problemorientierte Verbraucherpolitik. Der Beitrag der Verhaltensökonomik in einem verbesserten Verbraucherschutz, Sofia-Diskussionsbeiträge zur Institutionenanalyse Nr. 14-1, Darmstadt 2014.

34 Ebenda, S. 38 .

Title: Nudging in Public Policy - Good Intentions or Government Overreach?

Abstract: Behavioural economics and economic experiments can offer valuable insights to policymakers. Liberal or soft paternalism assumes that governmental nudging through the conscious setting of defaults in order to overcome potential behavioural anomalies can lead to better outcomes without giving up basic freedom of choice. Some authors recommend state interventions to prevent commercial firms from exploiting the effects of nudges, in particular through the use of defaults. They consider it necessary to create mandatory rules for how the nudging is permitted to be done. The state must decide on the scope and limits of the commitment power it grants to individuals. The danger of nudging is that information asymmetries can be used in order to manipulate people. The authors see nudges in the form of information provision and those for voluntarily improving self-control as useful. Educating individuals about the pitfalls of rational decision-making and using individual-level private mechanisms to avoid such pitfalls is put forward as a promising approach. Regarding paternalism as well as soft paternalism as enemies to freedom, liberal economists reject such state interference. JEL Classification: D03, D18, D60, D80 